\title{
EXISTENCE OF GUIDED CYLINDRICAL TM-MODES IN A HOMOGENEOUS SELF-FOCUSING DIELECTRIC
}

\author{
Charles A. STUART ${ }^{\mathrm{a}}$, Huan-Song ZHOU $^{\mathrm{b}}$ \\ ${ }^{a}$ Department of Mathematics, Ecole Polytechnique Fédérale de Lausanne, \\ CH-1015 Lausanne, Switzerland \\ ${ }^{\mathrm{b}}$ Young Scientist Laboratory of Mathematical Physics, \\ Wuhan Institute of Physics and Mathematics, Chinese Academy of Sciences, \\ P.O.Box 71010, Wuhan 430071, People's Republic of China
}

Received 21 February 2000

ABSTRACT. - We establish the existence of nontrivial solutions for the nonlinear eigenvalue problem which describes self-trapped transverse magnetic field modes in a cylindrical optical fiber made from a self-focusing dielectric material. It amounts to finding solutions in the Sobolev space $H_{0}^{1}(0, \infty)$ of a singular second order differential equation which is quasilinear and, in an appropriate sense, asymptotically linear. Solutions are critical points of an energy functional which has a mountain pass structure, although for all relevant parameter values the problem is in resonance at infinity. The quasilinearity complicates the proof of a Palais-Smale condition, and the asymptotic linearity means that the standard methods for showing that a P-S sequence is bounded do not apply. Both the linearization and the asymptotic linearization have only continuous spectrum. The eigenvalues determine the wavelengths of self-trapped modes and our results establish the existence of such modes for the largest possible range of wavelengths.

(C) 2001 L'Association Publications de l'Institut Henri Poincaré. Published by Elsevier B.V. All rights reserved

RÉSUMÉ. - On montre l'existence de solutions d'un problème aux valeurs propres non linéaire qui modélise des modes guidés cylindriques TM dans un fibre optique. Il s'agit de trouver des solutions dans l'espace de Sobolev $H_{0}^{1}(0, \infty)$ d'une équation différentielle singulière de deuxième ordre qui est quasilinéaire et asymptotiquement linéaire. Les solutions sont des points critiques d'une fonctionnelle ayant la structure du théorème du col mais qui se trouve dans un cas de résonance pour toutes les valeurs admises du paramètre. La forme quasilinéaire complique la vérification de la condition du Palais-Smale et le comportement à l'infini fait que les méthodes usuelles pour montrer que les suites P-S sont bornées ne s'appliquent pas. La linéarisation ainsi que la linéarisation à l'infini n'ont que du spectre continu. Les valeurs propres du problème non linéaire déterminent les longueurs d'onde des modes guidés et nos résultats établissent l'existence de tels modes pour la gamme maximum de longueurs d'ondes.

(C) 2001 L'Association Publications de l'Institut Henri Poincaré. Published by Elsevier B.V. All rights reserved

E-mail addresses: stuart@dma.epfl.ch (C.A. Stuart), hszhou@wipm.whcnc.ac.cn (H.S. Zhou). 


\section{Introduction}

The mathematical analysis of self-trapped beams of light propagating in a nonlinear dielectric medium is part of the study of special solutions of Maxwell's equations coupled with a nonlinear constitutive relation between the electric field and the electric displacement field. In modeling optical fibers it is natural to seek solutions having cylindrical symmetry. One family of special solutions describes situations where the electric field is everywhere perpendicular to the direction of propagation. For these transverse electric field (TE) modes the problem can be reduced to the study of a nonlinear second order differential equation with boundary conditions at infinity [5,9, 11] and [14]. A second (dual) family of special solutions corresponds to cases where the magnetic field is everywhere transverse to the direction of propagation $[2,3,8,12]$ and [13]. The equations governing these transverse magnetic field (TM) modes are more complicated than those for TE-modes because of the form of the constitutive relation in a nonlinear optical medium.

The discussion of guided waves in nonlinear optics is based on Maxwell's equations

$$
\begin{array}{ll}
\partial_{t} B=-c \nabla \wedge E \quad(1) & \partial_{t} D=c \nabla \wedge H \\
\nabla \cdot B=0 & \nabla \cdot D=0
\end{array}
$$

and the constitutive assumption for a homogeneous isotropic dielectric material is

$$
H=B \quad \text { (1) and } \quad D=\varepsilon\left(\left\langle E^{2}\right\rangle\right) E \quad \text { (2) }
$$

where $\varepsilon:[0, \infty) \rightarrow(0, \infty)$ is a given function and $\left\langle E^{2}\right\rangle$ denotes the time-average of the intensity $E \cdot E$ of the electric field. See $[12,13]$ and the many references therein to papers from physics and engineering. In a self-focusing material it is reasonable to suppose that the dielectric response function $\varepsilon$ has the following properties.

(A) $\varepsilon \in C([0, \infty)) \cap C^{1}((0, \infty))$ with $\varepsilon^{\prime}(s) \geqslant 0$ for $s>0, \varepsilon(0)>0$ and $\varepsilon(\infty)=$ $\lim _{s \rightarrow \infty} \varepsilon(s)<\infty$. Furthermore, $\lim _{s \rightarrow 0} s \varepsilon^{\prime}(s)=0$ and there exist $L>0$ and $\sigma>0$ such that

$$
\lim _{s \rightarrow 0} \frac{\varepsilon(s)-\varepsilon(0)}{s^{\sigma}}=L .
$$

In seeking travelling-wave solutions of (1.1) and (1.2) with cylindrical symmetry it is convenient to use cylindrical polar coordinates $(r, \theta, z)$ and to denote the usual curvilinear basis vectors by $i_{r}, i_{\theta}$ and $i_{z}$. In this notation an axi-symmetric TM-mode is a solution of (1.1) and (1.2) in which the magnetic field has the form

$$
B=u(r) \cos (k z-\omega t) i_{\theta},
$$

where $u:[0, \infty) \rightarrow \mathbb{R}$ is a smooth scalar function with

$$
u(0)=0
$$

to ensure the smoothness of $B$ at $r=0$. Then $|u(r)|, 2 \pi / k$ and $\omega$ give the amplitude, wavelength and frequency of the magnetic field. However, the system (1.1) and (1.2) 
does not reduce to an equation for the unknowns $u, k$ and $\omega$ in a straightforward way. It follows easily from (1.1)(2) and (1.2)(2) that if $B$ has the form (1.3) then there are smooth scalar functions $\varphi, \psi:[0, \infty) \rightarrow \mathbb{R}$ such that

$$
E=\varphi(r) \cos (k z-\omega t) i_{r}+\psi(r) \sin (k z-\omega t) i_{z}
$$

with

$$
\varphi(0)=0 \quad \text { and } \quad \psi^{\prime}(0)=0 .
$$

When studying TM-modes the standard procedure in nonlinear optics (see [2] and [3]) is to use $\varphi$ and $\psi$ as unknowns instead of $u$ because it is easy to reduce (1.1) and (1.2) to the following system for $\varphi$ and $\psi$,

$$
\begin{aligned}
& k\left(k \varphi+\psi^{\prime}\right)=\left(\frac{\omega}{c}\right)^{2} \varepsilon\left(\frac{1}{2}\left[\varphi^{2}+\psi^{2}\right]\right) \varphi, \\
& -\frac{1}{r}\left\{r\left(k \varphi+\psi^{\prime}\right)\right\}^{\prime}=\left(\frac{\omega}{c}\right)^{2} \varepsilon\left(\frac{1}{2}\left[\varphi^{2}+\psi^{2}\right]\right) \psi,
\end{aligned}
$$

where the prime denotes $\partial / \partial r$. The magnetic field corresponding to a solution of this system has the form (1.3) with $u=\left(\frac{c}{\omega}\right)\left(k \varphi+\psi^{\prime}\right)$. However, in more recent work [13], the problem has been reduced to a single equation for the function $u$. For this we introduce a new function, $\gamma:[0, \infty) \rightarrow(0, \infty)$, which is determined by $\varepsilon$ through the relation

$$
g(t) \equiv \gamma\left(\frac{1}{2} t^{2}\right) t
$$

where $g$ is the inverse of the function $f$ defined by $f(s)=\varepsilon\left(\frac{1}{2} s^{2}\right) s$. As is shown in Section 2.2 of [13], the properties $(A)$ of $\varepsilon$ ensure that $\gamma$ is well-defined and satisfies the following conditions $(H 1)$ to $(H 5)$.

(H1) $\gamma \in C([0, \infty)) \cap C^{1}((0, \infty))$.

(H2) For all $t>0, \gamma^{\prime}(t) \leqslant 0<\gamma(t)+2 t \gamma^{\prime}(t)$.

(H3) $\gamma(0)>0$ and $\gamma(\infty)=\lim _{t \rightarrow \infty} \gamma(t)>0$.

(H4) $\lim _{t \rightarrow 0} t \gamma^{\prime}(t)=0$.

(H5) There are constants $K>0$ and $\sigma>0$ such that

$$
\lim _{t \rightarrow 0} \frac{\gamma(t)-\gamma(0)}{t^{\sigma}}=-K
$$

With $\gamma$ as defined above there is a solution of (1.1) and (1.2) in which $B$ has the form (1.3) if and only if

$$
u(r)=\frac{\omega}{k c} w(k r),
$$

where $w:[0, \infty) \rightarrow \mathbb{R}$ satisfies the equation

$$
\left\{\gamma\left(\frac{1}{2}\left[w^{2}+\left(w^{\prime}+\frac{w}{r}\right)^{2}\right]\right)\left(w^{\prime}+\frac{w}{r}\right)\right\}^{\prime}-\gamma\left(\frac{1}{2}\left[w^{2}+\left(w^{\prime}+\frac{w}{r}\right)^{2}\right]\right) w+\lambda w=0
$$


with

$$
w(0)=0,
$$

where $\lambda=(\omega / k c)^{2}$.

A guided mode is a travelling-wave solution of (1.1) and (1.2) which has finite electromagnetic energy in planes which are perpendicular to the direction of propagation and in which all the fields decay to zero as $r \rightarrow \infty$. For solutions of the form (1.3) these guidance conditions become

$$
\int_{0}^{\infty}\left[w^{2}+\left(w^{\prime}\right)^{2}\right] r \mathrm{~d} r<\infty
$$

and

$$
\lim _{r \rightarrow \infty} w(r)=\lim _{r \rightarrow \infty} w^{\prime}(r)=0 .
$$

The power (total intensity) of the light beam described by such a solution is then given by

$$
P=\frac{\pi \omega}{k^{3}} \int_{0}^{\infty} \gamma\left(\frac{1}{2}\left[w^{2}+\left(w^{\prime}+\frac{w}{r}\right)^{2}\right]\right) w^{2} r \mathrm{~d} r
$$

All of these issues are discussed in more detail and the justified in [12] and [13] where it is also shown (see Proposition 3.1 of [13] and Corollary 3.5 of [12]) that the problem of finding solutions of (1.8) which satisfy the conditions (1.9), (1.10) and (1.11) is equivalent to finding

$$
(\lambda, z) \in(0, \infty) \times H_{0}^{1}(0, \infty) \quad \text { with } z \not \equiv 0
$$

such that

$$
\left\{a\left(r, z, z^{\prime}\right)\left[z^{\prime}+\frac{z}{2 r}\right]\right\}^{\prime}-a\left(r, z, z^{\prime}\right)\left\{z+\frac{1}{2 r}\left[z^{\prime}+\frac{z}{2 r}\right]\right\}+\lambda z=0 \quad \text { on }(0, \infty),
$$

where $z=z(r)=r^{1 / 2} w(r), z^{\prime}=z^{\prime}(r)$ and

$$
a(r, p, q)=\gamma\left(\frac{1}{2 r}\left[p^{2}+\left(q+\frac{p}{2 r}\right)^{2}\right]\right) \text { for } r>0 \text { and } p, q \in \mathbb{R} .
$$

Thus the existence of guided axi-symmetric TM-modes in a homogeneous medium is reduced to establishing the existence of solutions of (1.13) and (1.14) where $\gamma$ satisfies the conditions $(H 1)$ to $(H 5)$. This problem was studied in [12] where the following results are obtained concerning the existence of solutions.

(i) For $\lambda \notin(\gamma(\infty), \gamma(0)]$, there is no solution.

(ii) There exists $d_{0} \geqslant 0$ such that, for all $d>d_{0}$, there is a solution $\left(\lambda_{d}, z_{d}\right)$ of (1.14) with $\int_{0}^{\infty} z_{d}(r)^{2} \mathrm{~d} r=d$. Furthermore, $\lambda_{d} \in(\gamma(\infty), \gamma(0))$ and $z_{d}(r)>0$ for all $r>0$. Also $\lambda_{d} \rightarrow \gamma(\infty)$ as $d \rightarrow \infty$. 
(iii) If $0<\sigma<1$ in (H5), then we can set $d_{0}=0$ and we have $\lambda_{d} \rightarrow \gamma(0)$ as $d \rightarrow 0$.

(iv) If $\sigma \geqslant 1$ in (H5), then there exists $d_{1}>0$ such that (1.14) has no solution with $\int_{0}^{\infty} z_{d}(r)^{2} \mathrm{~d} r<d_{1}$.

In these results, the parameter $d \equiv \int_{0}^{\infty} z(r)^{2} \mathrm{~d} r$ is of physical interest since it is proportional to the total intensity of the light beam associated with the solution $z$. The existence results stated in (ii) and (iii) are obtained by using the condition $\int_{0}^{\infty} z(r)^{2} \mathrm{~d} r=$ $d$ as a constraint for the minimization of a functional defined on $H_{0}^{1}(0, \infty)$. In this approach the parameter $\lambda$ appears as Lagrange multiplier. Further results in this spirit concerning the existence of higher (nonpositive) modes are given in [9]. However the results (i) to (iv) do not give a complete picture of the situation concerning the existence of solutions of (1.14). For example, for $\sigma \geqslant 1$, they yield no information about the existence of solutions for values of $\lambda<\gamma(0)$ but near to $\gamma(0)$.

The main result of the present paper shows that all wavelengths within the interval $\left(\frac{2 \pi c}{\omega} \sqrt{\gamma(\infty)}, \frac{2 \pi c}{\omega} \sqrt{\gamma(0)}\right)$ are indeed possible, under the following mild additional assumption,

(H6) $\gamma^{\prime}(t)<0$ for all $t>0$ and $\lim _{t \rightarrow \infty} t \gamma^{\prime}(t)=0,\left(\Leftrightarrow \varepsilon^{\prime}(s)>0\right.$ for all $s>0$ and Then, $\left.\lim _{s \rightarrow \infty} s \varepsilon^{\prime}(s)=0\right)$.

(v) for any $\lambda \in(\gamma(\infty), \gamma(0))$, there exists a solution $(\lambda, z)$ of problem (1.14), and, by (i), we know that this conclusion is almost optimal. In fact, to ensure the existence of a solution with $\lambda=\gamma(0)$ requires extra hypotheses on the dielectric response.

Our method of proving (v) is to fix a value of $\lambda$ in the interval $(\gamma(\infty), \gamma(0))$ and to obtain a solution of (1.14) as a critical point of an appropriate energy functional, $J_{\lambda}$, by an application of a variant of the mountain pass theorem. In this approach there are two main difficulties which have to be overcome.

(a) The hypothesis (H3) implies that $J_{\lambda}$ has quadratic, but not super-quadratic, growth near infinity. This means that the usual ways, [7], of obtaining a bounded Palais-Smale sequence for $J_{\lambda}$ fail.

(b) In proving that $J_{\lambda}$ satisfies the Palais-Smale condition on $H_{0}^{1}(0, \infty)$, we have to confront simultaneously the problems due to the nonquadratic dependence of $J_{\lambda}$ on $z^{\prime}$ and the noncompactness of the Sobolev embeddings.

Some notation and preliminary results are given in Section 2. The functional, $J_{\lambda}$ defined on $H_{0}^{1}(0, \infty)$, which will be used is introduced in Section 3. In Section 4, we recall (as Proposition 4.2) a version of the mountain pass theorem, due to Bartolo, Benci and Fortunato [1], which is then used to prove our main result. The first two conditions which must be checked amount to verifying that the functional, $J_{\lambda}$, has what is usually called the mountain pass geometry. It is here that the restriction $\gamma(\infty)<\lambda<\gamma(0)$ appears in a natural way, but it should be observed that, for all such $\lambda$, the linearized problem at infinity is in resonance in the sense of [1] since the spectrum of that linearization (which is a form of Bessel's equation) is the whole interval $[\gamma(\infty), \infty)$. The remaining conditions, (c) and (d), correspond to showing that the condition (P-S)+ is satisfied for bounded Palais-Smale sequences and that some restricted Palais-Smale sequences are indeed bounded. In verifying (c), the noncompactness of the Sobolev embeddings on $(0, \infty)$ is compensated by the radial symmetry of the problem, so the real difficulty lies in dealing with the non-quadratic dependence of the functional, $J_{\lambda}$, 
on $z^{\prime}$. In this respect it is the inequality $\gamma(t)+2 t \gamma^{\prime}(t)>0$ in $(H 2)$ which allows us to obtain the required conclusion. This inequality asserts that $\gamma\left(\frac{1}{2} t^{2}\right) t$ is a strictly increasing function of $t$. Thus it is satisfied in every self-focusing medium. Our method of verifying the condition (d) was inspired by a recent paper by Jeanjean, [4], where a semilinear perturbation of the Laplacian with a similar quadratic growth of the energy functional at infinity is treated using a different variant of the mountain pass theorem. Our equation has a different and more complicated structure, but his approach to establishing the boundedness of a P-S sequence lies at the heart of our method of proving the condition (d).

Notation. In order to avoid lengthy expressions, we shall often truncate the argument of a function when we believe that no confusion should result. For example,

$$
\frac{1}{2 r}\left[z^{2}+\left(z^{\prime}+\frac{z}{2 r}\right)^{2}\right] \text { stands for } \frac{1}{2 r}\left[z(r)^{2}+\left(z^{\prime}(r)+\frac{z(r)}{2 r}\right)^{2}\right],
$$

where $z:(0, \infty) \rightarrow \mathbb{R}$, and

$$
a\left(r, z, z^{\prime}\right) \quad \text { stands for } a\left(r, z(r), z^{\prime}(r)\right) .
$$

\section{Notation and preliminary results}

In this section we establish some consequences of the hypotheses $(H 1)$ to $(H 6)$. We consider a function $\gamma$ satisfying $(H 1)$ and we set

$$
\begin{gathered}
\Gamma(t)=\int_{0}^{t} \gamma(s) \mathrm{d} s \quad \text { for } t \geqslant 0, \\
\tilde{\gamma}(t)=\gamma(t)-\gamma(0) \leqslant 0 \text { and } \tilde{\Gamma}(t)=\int_{0}^{t} \tilde{\gamma}(s) \mathrm{d} s \text { for } t \geqslant 0 .
\end{gathered}
$$

Clearly, $\tilde{\Gamma}(t)=\Gamma(t)-\gamma(0) t$ and, using $(H 2)$ and (H3), we see that

(i) $\Gamma \in C^{1}([0, \infty)) \cap C^{2}((0, \infty))$.

(ii) $\Gamma$ is strictly increasing and concave on $[0, \infty)$.

(iii) $0<\gamma(\infty) t \leqslant \gamma(t) t \leqslant \Gamma(t) \leqslant \gamma(0) t$ for $t>0$.

(iv) $-[\gamma(0)-\gamma(\infty)] t \leqslant \tilde{\Gamma}(t) \leqslant 0$ for $t \geqslant 0$.

We now deduce some further properties of these functions.

LEMMA 2.1. - Let $\gamma$ satisfy (H1) to (H3) and (H5). Then there is a constant $C>0$ such that

$$
|\tilde{\gamma}(t)| \leqslant C t^{\mu} \quad \text { for all } t \geqslant 0
$$

where $\mu=\min \{\sigma, 1\}$ and $\sigma$ is the constant appearing in (H5).

Proof. - By (H5), there exist constants $L>0$ and $\delta \in(0,1)$ such that

$$
|\tilde{\gamma}(t)| \leqslant L t^{\sigma} \leqslant L t^{\mu} \quad \text { for all } t \in[0, \delta) .
$$


Using (H2) and (H3), we also have that

$$
|\tilde{\gamma}(t)| \leqslant \gamma(0)-\gamma(\infty) \text { for all } t \geqslant 0 .
$$

Setting $C=\max \left\{L, \delta^{-\mu}[\gamma(0)-\gamma(\infty)]\right\}$ the result follows.

Next we consider the function $f:(0, \infty) \times \mathbb{R}^{2} \rightarrow \mathbb{R}$ defined by

$$
f(r, p, q)=r \Gamma\left(\frac{1}{2 r}\left[p^{2}+\left(q+\frac{p}{2 r}\right)^{2}\right]\right),
$$

where $\Gamma$ is given by (2.1). Clearly, $f \in C^{2}\left((0, \infty) \times \mathbb{R}^{2}\right)$ and we set

$$
g(r, p, q)=\partial_{p} f(r, p, q) \text { and } h(r, p, q)=\partial_{q} f(r, p, q) .
$$

Recalling (1.15), we have that

$$
\begin{aligned}
g(r, p, q) & =\gamma\left(\frac{1}{2 r}\left[p^{2}+\left(q+\frac{p}{2 r}\right)^{2}\right]\right)\left[p+\left(q+\frac{p}{2 r}\right) \frac{1}{2 r}\right] \\
& =a(r, p, q)\left[p+\left(q+\frac{p}{2 r}\right) \frac{1}{2 r}\right]
\end{aligned}
$$

and

$$
\begin{aligned}
h(r, p, q) & =\gamma\left(\frac{1}{2 r}\left[p^{2}+\left(q+\frac{p}{2 r}\right)^{2}\right]\right)\left(q+\frac{p}{2 r}\right) \\
& =a(r, p, q)\left(q+\frac{p}{2 r}\right) .
\end{aligned}
$$

Lemma 2.2. - Let $\gamma$ satisfy the conditions (H1) to (H4). Then for each $r>$ $0, h(r, \cdot) \in C^{1}\left(\mathbb{R}^{2}\right)$ with

$$
\begin{aligned}
& \text { (i) }\left|\partial_{p} h(r, p, q)\right| \leqslant\left(\frac{1}{2}+\frac{1}{r}\right) \gamma(0) \quad \text { and } \\
& \text { (ii) }\left|\partial_{q} h(r, p, q)\right| \leqslant 2 \gamma(0) .
\end{aligned}
$$

If (H6) is also satisfied, then there exists $\delta>0$ such that

$$
\partial_{q} h(r, p, q) \geqslant \delta \text { for all } r>0 \text { and }(p, q) \in \mathbb{R}^{2} .
$$

Proof. - For $r>0$ and $(p, q) \neq(0,0)$ we have that

$$
\partial_{p} h(r, p, q)=\gamma^{\prime}(\tau) \frac{1}{r}\left[p+\left(q+\frac{p}{2 r}\right) \frac{1}{2 r}\right]\left(q+\frac{p}{2 r}\right)+\frac{\gamma(\tau)}{2 r}
$$

and

$$
\partial_{q} h(r, p, q)=\gamma^{\prime}(\tau) \frac{1}{r}\left(q+\frac{p}{2 r}\right)^{2}+\gamma(\tau),
$$

where $\tau$ denotes the expression $\frac{1}{2 r}\left[p^{2}+\left(q+\frac{p}{2 r}\right)^{2}\right]$. Also for $r>0$,

$$
\partial_{p} h(r, 0,0)=\frac{\gamma(0)}{2 r} \quad \text { and } \quad \partial_{q} h(r, 0,0)=\gamma(0)
$$


since,

$$
\begin{gathered}
\frac{h(r, p, 0)-h(r, 0,0)}{p}=\frac{a(r, p, 0)}{2 r} \rightarrow \frac{\gamma(0)}{2 r} \quad \text { as } p \rightarrow 0, \\
\frac{h(r, 0, q)-h(r, 0,0)}{q}=a(r, 0, q) \rightarrow \gamma(0) \quad \text { as } p \rightarrow 0 .
\end{gathered}
$$

But, for $r>0$ and $(p, q) \neq(0,0)$, we have that

$$
\begin{aligned}
& \left|\gamma^{\prime}(\tau) \frac{1}{r}\left[p+\left(q+\frac{p}{2 r}\right) \frac{1}{2 r}\right]\left(q+\frac{p}{2 r}\right)\right| \\
& \quad \leqslant\left|\gamma^{\prime}(\tau)\right|\left\{\frac{1}{r}|p|\left|q+\frac{p}{2 r}\right|+\frac{1}{2 r^{2}}\left(q+\frac{p}{2 r}\right)^{2}\right\} \\
& \quad \leqslant\left|\gamma^{\prime}(\tau)\right|\left\{\frac{1}{2 r}\left[p^{2}+\left(q+\frac{p}{2 r}\right)^{2}\right]+\frac{1}{2 r^{2}}\left[p^{2}+\left(q+\frac{p}{2 r}\right)^{2}\right]\right\} \\
& \quad \leqslant\left(1+\frac{1}{r}\right)\left|\gamma^{\prime}(\tau)\right| \tau,
\end{aligned}
$$

and

$$
\left|\gamma^{\prime}(\tau) \frac{1}{r}\left(q+\frac{p}{2 r}\right)^{2}\right| \leqslant 2\left|\gamma^{\prime}(\tau)\right| \tau
$$

Using $(H 1)$ and $(H 4)$, it follows that $\partial_{p} h(r, \cdot)$ and $\partial_{q} h(r, \cdot)$ are continuous at $(p, q)=$ $(0,0)$ and so $h(r, \cdot) \in C^{1}\left(\mathbb{R}^{2}\right)$ for each $r>0$. Furthermore, for $r>0$ and $(p, q) \neq(0,0)$, we have

$$
\begin{aligned}
\left|\partial_{p} h(r, p, q)\right| & \leqslant-\left(1+\frac{1}{r}\right) \gamma^{\prime}(\tau) \tau+\frac{\gamma(\tau)}{2 r} \\
& \leqslant \frac{1}{2}\left(1+\frac{1}{r}\right) \gamma(\tau)+\frac{\gamma(\tau)}{2 r} \\
& =\left(\frac{1}{2}+\frac{1}{r}\right) \gamma(\tau) \leqslant\left(\frac{1}{2}+\frac{1}{r}\right) \gamma(0)
\end{aligned}
$$

and

$$
\left|\partial_{q} h(r, p, q)\right| \leqslant-2 \gamma^{\prime}(\tau) \tau+\gamma(\tau) \leqslant 2 \gamma(\tau) \leqslant 2 \gamma(0) .
$$

Finally we note that a function which also satisfies $(H 6)$ has the property that there is a $\delta>0$ such that $\gamma(t)+2 t \gamma^{\prime}(t) \geqslant \delta$. In fact, by $(H 3)$ and $(H 6), \lim _{t \rightarrow \infty} \gamma(t)+2 t \gamma^{\prime}(t)=$ $\gamma(\infty)>0$, whereas, by $(H 3)$ and $(H 4), \lim _{t \rightarrow 0} \gamma(t)+2 t \gamma^{\prime}(t)=\gamma(0)>0$. Using $(H 2)$ it now follows that $\inf _{t>0} \gamma(t)+2 t \gamma^{\prime}(t)>0$ as claimed. Hence, for $r>0$ and $(p, q) \neq(0,0)$, we have

$$
\partial_{q} h(r, p, q) \geqslant 2 \gamma^{\prime}(\tau) \tau+\gamma(\tau) \geqslant \delta
$$




\section{The variational problem}

In this section we formulate the variational problem which will be used to deal with (1.14). We begin by recalling some essential properties of the Sobolev space $H_{0}^{1}(0, \infty)$. It is a real Hilbert space with the norm defined by

$$
\|z\|=\left\{\int_{0}^{\infty}\left\{z(r)^{2}+\left|z^{\prime}(r)\right|^{2}\right\} \mathrm{d} r\right\}^{1 / 2} .
$$

The usual norm on the Banach space $L^{p}(0, \infty)$ will be denoted by $|z|_{p}$ for $1 \leqslant p \leqslant \infty$. For any $z(r) \in H_{0}^{1}(0, \infty)$, the following estimates are valid,

$$
\begin{gathered}
|z(r)|_{\infty}^{2} \leqslant 2|z(r)|_{2}\left|z^{\prime}(r)\right|_{2}, \\
|z(r)| \leqslant r^{1 / 2}\left|z^{\prime}(r)\right|_{2},
\end{gathered}
$$

and

$$
\int_{0}^{\infty} \frac{z(r)^{2}}{r^{2}} \mathrm{~d} r \leqslant 4 \int_{0}^{\infty} z^{\prime}(r)^{2} \mathrm{~d} r
$$

Furthermore,

$$
\lim _{r \rightarrow 0} r^{-1 / 2} z(r)=\lim _{r \rightarrow \infty} z(r)=0 .
$$

LEMMA 3.1. - For any $z \in H_{0}^{1}(0, \infty)$,

$$
\int_{0}^{\infty}\left(z^{\prime}+\frac{z}{2 r}\right)^{2} \mathrm{~d} r=\int_{0}^{\infty}\left(z^{\prime}\right)^{2}+\frac{3 z^{2}}{4 r^{2}} \mathrm{~d} r .
$$

Hence the expression

$$
\|z\|_{1}=\left\{\int_{0}^{\infty} z^{2}+\left(z^{\prime}+\frac{z}{2 r}\right)^{2} \mathrm{~d} r\right\}^{1 / 2}
$$

defines a norm on $H_{0}^{1}(0, \infty)$ which is equivalent to that defined by (3.1).

$$
\begin{aligned}
& \text { Proof. - For } z \in H_{0}^{1}(0, \infty) \text { and } 0<a<b<\infty \\
& \qquad \int_{a}^{b} \frac{z^{\prime}(r) z(r)}{r} \mathrm{~d} r=\frac{1}{2} \int_{a}^{b} \frac{\left\{z(r)^{2}\right\}^{\prime}}{r} \mathrm{~d} r=\frac{1}{2}\left\{\frac{z(b)^{2}}{b}-\frac{z(a)^{2}}{a}+\int_{a}^{b} \frac{z(r)^{2}}{r^{2}} \mathrm{~d} r\right\}
\end{aligned}
$$

and so, by (3.4) and (3.5),

$$
\int_{0}^{\infty} \frac{z^{\prime}(r) z(r)}{r} \mathrm{~d} r=\frac{1}{2} \int_{0}^{\infty} \frac{z(r)^{2}}{r^{2}} \mathrm{~d} r .
$$


This proves (3.6), and (3.7) follows easily since

$$
\|z\|^{2} \leqslant \int_{0}^{\infty}\left(z^{\prime}\right)^{2}+z^{2}+\frac{3 z^{2}}{4 r^{2}} \mathrm{~d} r=\|z\|_{1}^{2} \leqslant \int_{0}^{\infty} 4\left(z^{\prime}\right)^{2}+z^{2} \mathrm{~d} r \leqslant 4\|z\|^{2} \quad \text { by (3.4). }
$$

We now introduce the function $J$ on $H_{0}^{1}(0, \infty)$ which will be used to deal with (1.14). For a number $\lambda$ and a function $\gamma$ which satisfies $(H 1)$ to $(H 3)$, we set

$$
J(z)=\int_{0}^{\infty} r \Gamma\left(\frac{1}{2 r}\left[z^{2}+\left(z^{\prime}+\frac{z}{2 r}\right)^{2}\right]\right) \mathrm{d} r-\frac{\lambda}{2} \int_{0}^{\infty} z^{2} \mathrm{~d} r
$$

where $\Gamma$ is defined by (2.1) and $z \in H_{0}^{1}(0, \infty)$.

Since $\gamma(\infty) t \leqslant \Gamma(t) \leqslant \gamma(0) t$ for all $t \geqslant 0$, it is easy to see that

$$
-\infty<\frac{1}{2}\left\{\gamma(\infty)\|z\|_{1}^{2}-\lambda|z|_{2}^{2}\right\} \leqslant J(z) \leqslant \frac{1}{2}\left\{\gamma(0)\|z\|_{1}^{2}-\lambda|z|_{2}^{2}\right\}<\infty
$$

for all $z \in H_{0}^{1}(0, \infty)$.

In fact, $J$ is continuously differentiable on $H_{0}^{1}(0, \infty)$ and any nonzero critical point of $J$ is a solution of (1.14). More precisely, we have the following results.

LEMMA 3.2. - Let $\gamma$ satisfy the conditions (H1) to (H5). Then

(i) For any $\lambda \in \mathbb{R}, J \in C^{1}\left(H_{0}^{1}(0, \infty), \mathbb{R}\right)$ with

$$
J^{\prime}(z) \varphi=\int_{0}^{\infty} a\left(r, z, z^{\prime}\right)\left\{z \varphi+\left(z^{\prime}+\frac{z}{2 r}\right)\left(\varphi^{\prime}+\frac{\varphi}{2 r}\right)\right\}-\lambda z \varphi \mathrm{d} r
$$

for all $z, \varphi \in H_{0}^{1}(0, \infty)$.

(ii) If $\lambda \in \mathbb{R}$ and $z \in H_{0}^{1}(0, \infty)$ are such that

$$
J^{\prime}(z) \varphi=0 \quad \text { for all } \varphi \in H_{0}^{1}(0, \infty),
$$

then $z \in C^{2}(0, \infty)$ and $(\lambda, z)$ satisfies (1.14).

Proof. - Part (i) is Lemma 3.1 of [12] and part (ii) is Theorem 3.3 of [12].

Remark. - Inspecting the proofs of the results cited from [12], we see that Lemma 3.2 is valid even if $\gamma$ only satisfies $(H 1)$ to $(H 4)$.

We end this section with two technical results, which will be used later.

Lemma 3.3. - Let $\gamma$ satisfy the conditions $(H 1)$ to $(H 3)$, let $\left\{u_{n}\right\} \subset H_{0}^{1}(0, \infty)$ be bounded and set

$$
\begin{aligned}
& v_{n}(r)=\int_{0}^{r} s g\left(s, u_{n}(s), u_{n}^{\prime}(s)\right) \mathrm{d} s \quad \text { and } \\
& V_{n}(r)=\int_{0}^{r} h\left(s, u_{n}(s), u_{n}^{\prime}(s)\right) \mathrm{d} s,
\end{aligned}
$$


where $g$ and $h$ are defined by (2.4) and (2.5), respectively. Then by passing to a subsequence which we still denote by $\left\{u_{n}\right\}$, we can assume that the sequences $\left\{v_{n}(r)\right\}$ and $\left\{V_{n}(r)\right\}$ converge strongly in $L^{2}(0, b)$ for all $b \in(0, \infty)$.

Proof. - Let $K>0$ be such that $\left\|u_{n}\right\| \leqslant K$ for all $n \in \mathbb{N}$. By (3.2)

$$
\left|u_{n}\right|_{\infty} \leqslant K, \quad\left|u_{n}\right|_{2} \leqslant K \quad \text { and } \quad\left|u_{n}^{\prime}\right|_{2} \leqslant K .
$$

The function $h\left(s, u_{n}(s), u_{n}^{\prime}(s)\right)$ is integrable on $(0, r)$ since

$$
\left|h\left(s, u_{n}(s), u_{n}^{\prime}(s)\right)\right| \leqslant \gamma(0)\left|u_{n}^{\prime}(s)+\frac{u_{n}(s)}{2 s}\right|
$$

and

$$
\begin{aligned}
\int_{0}^{r}\left|h\left(s, u_{n}(s), u_{n}^{\prime}(s)\right)\right| \mathrm{d} s & \leqslant \gamma(0) r^{1 / 2}\left\{\int_{0}^{r}\left|u_{n}^{\prime}(s)+\frac{u_{n}(s)}{2 s}\right|^{2} \mathrm{~d} s\right\}^{1 / 2} \\
& \leqslant 2 \gamma(0) r^{1 / 2}\left|u_{n}^{\prime}\right|_{2} \leqslant 2 K \gamma(0) r^{1 / 2} .
\end{aligned}
$$

Hence $V_{n}$ has a weak derivative on $(0, \infty)$ and

$$
V_{n}^{\prime}(r)=h\left(r, u_{n}(r), u_{n}^{\prime}(r)\right) \quad \text { a.e. on }(0, \infty) .
$$

Furthermore, by (3.4) and (3.6) we have

$$
\int_{0}^{\infty}\left|V_{n}^{\prime}(r)\right|^{2} \mathrm{~d} r \leqslant \gamma(0)^{2} \int_{0}^{\infty}\left(u_{n}^{\prime}(r)+\frac{u_{n}(r)}{2 r}\right)^{2} \mathrm{~d} r \leqslant 4 \gamma(0)^{2} K^{2} .
$$

By (3.12) and (3.13) it follows that $\left\{V_{n}(r)\right\}$ is a bounded sequence in $H^{1}(0, b)$ for any $b>0$. Hence passing to a suitable (diagonal) subsequence we may suppose that $\left\{V_{n}(r)\right\}$ converges strongly in $L^{2}(0, b)$ for every $b>0$.

The function $s g\left(s, u_{n}(s), u_{n}^{\prime}(s)\right)$ is also integrable on $(0, r)$ since

$$
s\left|g\left(s, u_{n}(s), u_{n}^{\prime}(s)\right)\right| \leqslant \gamma(0)\left\{s\left|u_{n}(s)\right|+\frac{1}{2}\left|u_{n}^{\prime}(s)+\frac{u_{n}(s)}{2 s}\right|\right\}
$$

and

$$
\begin{aligned}
\int_{0}^{r} s\left|g\left(s, u_{n}(s), u_{n}^{\prime}(s)\right)\right| \mathrm{d} s & \leqslant \gamma(0)\left\{\frac{r^{3 / 2}}{\sqrt{3}}\left|u_{n}\right|_{2}+r^{1 / 2}\left|u_{n}^{\prime}\right|_{2}\right\} \\
& \leqslant K \gamma(0) r^{1 / 2}\left\{\frac{r}{\sqrt{3}}+1\right\} \quad \text { by (3.4) and (3.6). }
\end{aligned}
$$

Hence $v_{n}$ has a weak derivative on $(0, \infty)$ and

$$
v_{n}^{\prime}(r)=r g\left(r, u_{n}(r), u_{n}^{\prime}(r)\right) \quad \text { a.e. on }(0, \infty) .
$$

Thus, for any $b>0$, we have that 


$$
\begin{aligned}
\int_{0}^{b} v_{n}^{\prime}(r)^{2} \mathrm{~d} r & \leqslant \gamma(0)^{2} \int_{0}^{b}\left\{r u_{n}(r)+\frac{1}{2}\left[u_{n}^{\prime}(r)+\frac{u_{n}(r)}{2 r}\right]\right\}^{2} \mathrm{~d} r \\
& \leqslant 2 \gamma(0)^{2} \int_{0}^{b}\left\{r^{2} u_{n}(r)^{2}+\frac{1}{4}\left[u_{n}^{\prime}(r)+\frac{u_{n}(r)}{2 r}\right]^{2}\right\} \mathrm{d} r \\
& \leqslant 2 \gamma(0)^{2}\left\{b^{2}\left|u_{n}\right|_{2}^{2}+\left|u_{n}^{\prime}\right|_{2}^{2}\right\} \quad \text { by (3.4) and (3.6) } \\
& \leqslant 2 \gamma(0)^{2}\left(b^{2}+1\right) K^{2} .
\end{aligned}
$$

Therefore, $\left\{v_{n}(r)\right\}$ is a bounded sequence in $H^{1}(0, b)$ for any $b>0$ and so, by passing to a further (diagonal) sequence, we may assume that $\left\{v_{n}(r)\right\}$ converges strongly in $L^{2}(0, b)$ for every $b>0$.

Finally we recall the following result which will be used several times.

LEMMA 3.4 ([10], Proposition 3.4). - Let $\left\{u_{n}\right\}$ be a bounded sequence in $L^{p}(0, \infty)$ for some $p \in(1, \infty)$ such that $u_{n}(r) \stackrel{n}{\rightarrow} u(r)$ for almost all $r>0$. Then $u \in L^{p}(0, \infty)$ and $u_{n} \stackrel{n}{\rightarrow} u$ weakly in $L^{p}(0, \infty)$.

\section{The main result}

We now come to the main result of this paper.

THEOREM 4.1. - Let $\gamma$ satisfy the conditions (H1) to (H6) and let $\lambda \in(\gamma(\infty)$, $\gamma(0))$. Then there exists $z(r) \in C^{2}((0, \infty)) \cap H_{0}^{1}(0, \infty)$ such that $z \not \equiv 0$ and $(\lambda, z)$ satisfies Eq. (1.14).

In view of Lemma 3.2(ii), it is enough to show that, under the hypotheses of Theorem 4.1, the functional $J: H_{0}^{1}(0, \infty) \rightarrow \mathbb{R}$ defined by (3.8) satisfies the conditions which are required for the following version of the mountain pass theorem.

PROPOSITION 4.2 (Bartolo, Benci and Fortunato [1]). - Let H be a real Hilbert space and consider a functional $J \in C^{1}(H, \mathbb{R})$ which satisfies the following conditions.

(a) $J(0)=0$ and there exist $\rho>0$ and $\alpha>0$ such that $J(u) \geqslant \alpha$ for all $u \in H$ with $\|u\|=\rho$.

(b) There exists $e \in H$ with $\|e\|>\rho$ such that $J(e) \leqslant 0$.

(c) For any $c>0$, every bounded sequence $\left\{u_{n}\right\}$ in $H$ such that

$$
J\left(u_{n}\right) \stackrel{n}{\rightarrow} c \quad \text { and } \quad J^{\prime}\left(u_{n}\right) \stackrel{n}{\rightarrow} 0 \text { strongly in } H^{*}
$$

possesses a convergent subsequence.

(d) For any $c>0$, there exist positive constants $\delta, R$ and $\eta$ such that

$$
\left\|J^{\prime}(u)\right\|\|u\| \geqslant \eta \quad \text { for all } u \in A,
$$

where $A=\{u \in H: c-\delta \leqslant J(u) \leqslant c+\delta$ and $\|u\| \geqslant R\}$.

Then, there exists an element $u \in H$ such that $J(u) \geqslant \alpha$ and $J^{\prime}(u)=0$. 
We prove Theorem 4.1 by showing that the functional $J$, defined by (3.8) for a fixed value of $\lambda \in(\gamma(\infty), \gamma(0))$, satisfies the hypotheses of Proposition 4.2. Clearly, $J(0)=0$ and by Lemma 3.2(i) we have that $J \in C^{1}\left(H_{0}^{1}(0, \infty), \mathbb{R}\right)$.

Verification of (a). The function $\Gamma$ is concave on $[0, \infty)$ and so

$$
\begin{aligned}
\Gamma\left(\frac{1}{2 r}\left[z^{2}+\left(z^{\prime}+\frac{z}{2 r}\right)^{2}\right]\right) & \geqslant \frac{1}{2} \Gamma\left(\frac{z^{2}}{r}\right)+\frac{1}{2} \Gamma\left(\frac{1}{r}\left(z^{\prime}+\frac{z}{2 r}\right)^{2}\right) \\
& \geqslant \frac{1}{2} \Gamma\left(\frac{z^{2}}{r}\right)+\frac{\gamma(\infty)}{2 r}\left(z^{\prime}+\frac{z}{2 r}\right)^{2}
\end{aligned}
$$

for all $r>0$ and $z \in H_{0}^{1}(0, \infty)$, since $\Gamma(t) \geqslant \gamma(\infty) t$ for all $t \geqslant 0$. Thus, for any $z \in H_{0}^{1}(0, \infty)$,

$$
\begin{aligned}
J(z) & \geqslant \frac{\gamma(\infty)}{2} \int_{0}^{\infty}\left(z^{\prime}+\frac{z}{2 r}\right)^{2} \mathrm{~d} r+\frac{1}{2} \int_{0}^{\infty}\left\{r \Gamma\left(\frac{z^{2}}{r}\right)-\lambda z^{2}\right\} \mathrm{d} r \\
& \geqslant \frac{\gamma(\infty)}{2}\left|z^{\prime}\right|_{2}^{2}+\frac{1}{2} \int_{0}^{\infty}\left\{r \Gamma\left(\frac{z^{2}}{r}\right)-\lambda z^{2}\right\} \mathrm{d} r \quad \text { by (3.6). }
\end{aligned}
$$

Set $\varepsilon=(\gamma(0)-\lambda) / 2$. Then $\varepsilon>0$ and there exists $\delta>0$ such that

$$
\gamma(t) \geqslant \gamma(0)-\varepsilon \quad \text { for all } t \in[0, \delta]
$$

and consequently,

$$
\Gamma(t) \geqslant(\gamma(0)-\varepsilon) t \quad \text { for all } t \in[0, \delta] .
$$

By (3.3), $z^{2} / r \leqslant\left|z^{\prime}\right|_{2}^{2} \leqslant\|z\|^{2}$ and so

$$
\Gamma\left(\frac{z^{2}}{r}\right) \geqslant(\gamma(0)-\varepsilon) \frac{z^{2}}{r} \quad \text { for all } z \in H_{0}^{1}(0, \infty) \text { such that }\|z\|^{2} \leqslant \delta .
$$

Hence for $z \in H_{0}^{1}(0, \infty)$ with $\|z\|^{2} \leqslant \delta$,

$$
\begin{aligned}
J(z) & \geqslant \frac{\gamma(\infty)}{2}\left|z^{\prime}\right|_{2}^{2}+\frac{1}{2} \int_{0}^{\infty}\left[(\gamma(0)-\varepsilon) z^{2}-\lambda z^{2}\right] \mathrm{d} r \\
& =\frac{\gamma(\infty)}{2}\left|z^{\prime}\right|_{2}^{2}+\frac{\gamma(0)-\lambda}{4}|z|_{2}^{2} \\
& \geqslant \frac{1}{2} \min \left\{\gamma(\infty), \frac{\gamma(0)-\lambda}{2}\right\}\|z\|^{2} .
\end{aligned}
$$

Setting $\rho(\lambda)=\sqrt{\delta}$ and $\alpha(\lambda)=\delta / 2 \min \{\gamma(\infty),(\gamma(0)-\lambda) / 2\}$, we see that the condition (a) is satisfied.

Verification of (b). For $\alpha>0$, we set

$$
v_{\alpha}(r)=2 \alpha^{3 / 2} r \mathrm{e}^{-\alpha r}, \quad \text { for } r \geqslant 0 .
$$


Then for all $\alpha>0, v_{\alpha} \in H_{0}^{1}(0, \infty)$ with $\left|v_{\alpha}\right|_{2}=1$ and $\left|v_{\alpha}^{\prime}\right|_{2}=\alpha$. Hence for $\alpha, t>0$,

$$
\frac{J\left(t v_{\alpha}\right)}{t^{2}}=\frac{1}{t^{2}} \int_{0}^{\infty} r \Gamma\left(\frac{t^{2}}{2 r}\left[v_{\alpha}^{2}+\left(v_{\alpha}^{\prime}+\frac{v_{\alpha}}{2 r}\right)^{2}\right]\right) \mathrm{d} r-\frac{\lambda}{2}\left|v_{\alpha}\right|_{2}^{2}
$$

and, for $r>0$,

$$
\begin{aligned}
& \frac{1}{t^{2}} \Gamma\left(\frac{t^{2}}{2 r}\left[v_{\alpha}(r)^{2}+\left(v_{\alpha}^{\prime}(r)+\frac{v_{\alpha}(r)}{2 r}\right)^{2}\right]\right) \\
& \quad=\frac{1}{t^{2}} \int_{0}^{\frac{t^{2}}{2 r}\left[v_{\alpha}(r)^{2}+\left(v_{\alpha}^{\prime}(r)+\frac{v_{\alpha}(r)}{2 r}\right)^{2}\right]} \gamma(s) \mathrm{d} s \\
& \quad=\int_{0}^{1} \gamma\left(\frac{t^{2}}{2 r}\left[v_{\alpha}(r)^{2}+\left(v_{\alpha}^{\prime}(r)+\frac{v_{\alpha}(r)}{2 r}\right)^{2}\right] \tau\right) \frac{1}{2 r}\left[v_{\alpha}(r)^{2}+\left(v_{\alpha}^{\prime}(r)+\frac{v_{\alpha}(r)}{2 r}\right)^{2}\right] \mathrm{d} \tau \\
& \quad \rightarrow \frac{1}{2 r}\left[v_{\alpha}(r)^{2}+\left(v_{\alpha}^{\prime}(r)+\frac{v_{\alpha}(r)}{2 r}\right)^{2}\right] \gamma(\infty) \text { as } t \rightarrow \infty
\end{aligned}
$$

by dominated convergence.

Hence, again by dominated convergence, for all $\alpha>0$,

$$
\begin{aligned}
\lim _{t \rightarrow \infty} \frac{J\left(t v_{\alpha}\right)}{t^{2}} & =\frac{\gamma(\infty)}{2} \int_{0}^{\infty}\left[v_{\alpha}^{2}+\left(v_{\alpha}^{\prime}+\frac{v_{\alpha}}{2 r}\right)^{2}\right] \mathrm{d} r-\frac{\lambda}{2} \\
& =\frac{\gamma(\infty)}{2}\left[1+\alpha^{2}+\frac{3}{4} \int_{0}^{\infty} \frac{v_{\alpha}^{2}}{r^{2}} \mathrm{~d} r\right]-\frac{\lambda}{2} \\
& =\frac{\gamma(\infty)}{2}\left[1+\frac{5}{2} \alpha^{2}\right]-\frac{\lambda}{2} .
\end{aligned}
$$

Choosing $\alpha=\frac{1}{2} \sqrt{(\lambda-\gamma(\infty)) /(5 \gamma(\infty))}$ we have that

$$
\lim _{t \rightarrow \infty} \frac{J\left(t v_{\alpha}\right)}{t^{2}}<0
$$

and there exists $T=T(\alpha)>0$ such that $J\left(t v_{\alpha}\right) \leqslant 0$ for all $t \geqslant T$. But $\left\|t v_{\alpha}\right\|=$ $t\left\{1+\alpha^{2}\right\}^{1 / 2}$ and so by choosing

$$
t=\max \left\{T, \frac{2 \rho}{\left(1+\alpha^{2}\right)^{1 / 2}}\right\},
$$

where $\rho$ is chosen as in (a), we see that (b) is satisfied by $e=t v_{\alpha}$.

Verification of (c). We fix $c>0$ and consider a bounded sequence $\left\{u_{n}\right\}$ in $H_{0}^{1}(0, \infty)$ such that

$$
J^{\prime}\left(u_{n}\right) \stackrel{n}{\rightarrow} 0 \quad \text { strongly in } H^{-1}(0, \infty)
$$


By passing to a subsequence, we may suppose that there is an element $u \in H_{0}^{1}(0, \infty)$ such that

$$
\begin{gathered}
u_{n} \stackrel{n}{\rightarrow} u \quad \text { weakly in } H_{0}^{1}(0, \infty), \\
u_{n} \stackrel{n}{\rightarrow} u \quad \text { strongly in } L^{2}(0, b) \text { for every } b>0, \\
u_{n}(r) \stackrel{n}{\rightarrow} u(r) \quad \text { a.e. on }(0, \infty) .
\end{gathered}
$$

Step 1. The first step is to show that we may also assume that

(i) $u_{n}^{\prime} \stackrel{n}{\rightarrow} u^{\prime}$ strongly in $L^{2}(a, b)$ for all $a, b \in(0, \infty)$ with $a<b$, and (ii)

$$
u_{n}^{\prime} \stackrel{n}{\rightarrow} u^{\prime} \quad \text { a.e. on }(0, \infty) .
$$

Using the Riesz representation theorem on $H_{0}^{1}(0, \infty)$ with the scalar product

$$
\langle u, v\rangle=\int_{0}^{\infty}\left\{u^{\prime} v^{\prime}+u v\right\} \mathrm{d} r \quad \text { for } u, v \in H_{0}^{1}(0, \infty),
$$

there exists an element $\eta_{n}(r) \in H_{0}^{1}(0, \infty)$ such that

$$
J^{\prime}\left(u_{n}\right) \varphi=\left\langle\eta_{n}, \varphi\right\rangle \quad \text { for all } \varphi \in H_{0}^{1}(0, \infty),
$$

and, by (4.1),

$$
\left\|\eta_{n}\right\| \stackrel{n}{\rightarrow} 0
$$

Recalling the expression (3.9) for $J^{\prime}$, this means that

$$
\begin{gathered}
\int_{0}^{\infty} a\left(r, u_{n}, u_{n}^{\prime}\right)\left\{u_{n} \varphi+\left(u_{n}^{\prime}+\frac{u_{n}}{2 r}\right)\left(\varphi^{\prime}+\frac{\varphi}{2 r}\right)\right\}-\lambda u_{n} \varphi \mathrm{d} r \\
=\int_{0}^{\infty} \eta_{n}^{\prime} \varphi^{\prime}+\eta_{n} \varphi \mathrm{d} r \quad \text { for all } \varphi \in H_{0}^{1}(0, \infty)
\end{gathered}
$$

which can be rewritten as

$$
\begin{aligned}
& \int_{0}^{\infty}\left[h\left(r, u_{n}, u_{n}^{\prime}\right)-\eta_{n}^{\prime}\right] \varphi^{\prime} \mathrm{d} r+\int_{0}^{\infty}\left[g\left(r, u_{n}, u_{n}^{\prime}\right)-\eta_{n}-\lambda u_{n}\right] \varphi \mathrm{d} r=0 \\
& \quad \text { for all } \varphi \in H_{0}^{1}(0, \infty),
\end{aligned}
$$

in the notation (2.4) and (2.5). Setting

$$
\begin{aligned}
& w_{n}(r)=h\left(r, u_{n}(r), u_{n}^{\prime}(r)\right)-\eta_{n}^{\prime}(r) \quad \text { and } \\
& z_{n}(r)=g\left(r, u_{n}(r), u_{n}^{\prime}(r)\right)-\eta_{n}(r)-\lambda u_{n}(r),
\end{aligned}
$$

the functions $w_{n}$ and $z_{n}$ are locally integrable on $(0, \infty)$ and, by (4.7), $w_{n}$ has a weak derivative on $(0, \infty)$ where 


$$
w_{n}^{\prime}(r)=z_{n}(r) \quad \text { a.e. on }(0, \infty) .
$$

In particular, $w_{n}$ is continuous on $(0, \infty)$ (after modification on a set of measure zero) and for any $a>0$,

$$
\int_{a}^{r} s z_{n}(s) \mathrm{d} s=\int_{a}^{r} s w_{n}^{\prime}(s) \mathrm{d} s=r w_{n}(r)-a w_{n}(a)-\int_{a}^{r} w_{n}(s) \mathrm{d} s .
$$

Thus for $a, r>0$,

$$
a w_{n}(a)=r w_{n}(r)-\int_{a}^{r} w_{n}(s) \mathrm{d} s-\int_{a}^{r} s z_{n}(s) \mathrm{d} s .
$$

In the proof of Lemma 3.3 we showed that $h\left(s, u_{n}(s), u_{n}^{\prime}(s)\right)$ and $s g\left(s, u_{n}(s), u_{n}^{\prime}(s)\right)$ are both integrable on $(0, r)$ for every $r>0$. From this and (4.9) it follows that $\lim _{a \rightarrow 0} a w_{n}(a)$ exists and that

$$
\lim _{a \rightarrow 0} a w_{n}(a)=r w_{n}(r)+\eta_{n}(r)-V_{n}(r)-v_{n}(r)+\int_{0}^{r} s\left\{\lambda u_{n}(s)+\eta_{n}(s)\right\} \mathrm{d} s,
$$

where we have used the notation (3.10).

But

$$
w_{n}(r)=V_{n}^{\prime}(r)-\eta_{n}^{\prime}(r) \quad \text { a.e. on }(0, \infty)
$$

and so, by (3.13), $w_{n} \in L^{2}(0, \infty)$.

Thus we must have that

$$
\lim _{a \rightarrow 0} a w_{n}(a)=0
$$

and so (4.10) yields

$$
w_{n}(r)=\frac{1}{r}\left\{V_{n}(r)+v_{n}(r)-\eta_{n}(r)-\int_{0}^{r} s\left[\eta_{n}(s)+\lambda u_{n}(s)\right] \mathrm{d} s\right\} .
$$

Now for $r>0, h(r, \cdot) \in C^{1}\left(\mathbb{R}^{2}\right)$ by Lemma 2.2. Hence for any $n, m \geqslant 1$,

$$
\begin{aligned}
& h\left(r, u_{n}(r), u_{n}^{\prime}(r)\right)-h\left(r, u_{m}(r), u_{m}^{\prime}(r)\right) \\
& =\int_{0}^{1} \frac{\mathrm{d}}{\mathrm{d} t} h\left(r, t u_{n}(r)+(1-t) u_{m}(r), t u_{n}^{\prime}(r)+(1-t) u_{m}^{\prime}(r)\right) \mathrm{d} t \\
& =\int_{0}^{1} A(t, r, n, m)\left[u_{n}(r)-u_{m}(r)\right]+B(t, r, n, m)\left[u_{n}^{\prime}(r)-u_{m}^{\prime}(r)\right] \mathrm{d} t,
\end{aligned}
$$

where

$$
A(t, r, n, m)=\partial_{p} h\left(r, t u_{n}(r)+(1-t) u_{m}(r), t u_{n}^{\prime}(r)+(1-t) u_{m}^{\prime}(r)\right)
$$


and

$$
B(t, r, n, m)=\partial_{q} h\left(r, t u_{n}(r)+(1-t) u_{m}(r), t u_{n}^{\prime}(r)+(1-t) u_{m}^{\prime}(r)\right) .
$$

Using the mean-value theorem for integrals, there exists $\theta=\theta(r, n, m) \in(0,1)$ such that

$$
\begin{aligned}
& h\left(r, u_{n}(r), u_{n}^{\prime}(r)\right)-h\left(r, u_{m}(r), u_{m}^{\prime}(r)\right) \\
& =\partial_{p} h(r, p(r, n, m), q(r, n, m))\left(u_{n}-u_{m}\right)(r) \\
& \quad+\partial_{q} h(r, p(r, n, m), q(r, n, m))\left(u_{n}^{\prime}-u_{m}^{\prime}\right)(r),
\end{aligned}
$$

where $p(r, n, m)=\theta u_{n}(r)+(1-\theta) u_{m}(r)$ and $q(r, n, m)=\theta u_{n}^{\prime}(r)+(1-\theta) u_{m}^{\prime}(r)$.

Using (4.12) and Lemma 2.2, there exists $\delta>0$ such that

$$
\begin{aligned}
\delta\left|u_{n}^{\prime}(r)-u_{m}^{\prime}(r)\right| \\
\quad \leqslant \partial_{q} h(r, p(r, n, m), q(r, n, m))\left|u_{n}^{\prime}(r)-u_{m}^{\prime}(r)\right| \\
\quad \leqslant\left(\frac{1}{2}+\frac{1}{r}\right) \gamma(0)\left|u_{n}(r)-u_{m}(r)\right|+\left|h\left(r, u_{n}(r), u_{n}^{\prime}(r)\right)-h\left(r, u_{m}(r), u_{m}^{\prime}(r)\right)\right| \\
\quad \leqslant\left(\frac{1}{2}+\frac{1}{r}\right) \gamma(0)\left|u_{n}(r)-u_{m}(r)\right|+\left|\eta_{n}^{\prime}(r)-\eta_{m}^{\prime}(r)\right|+\left|w_{n}(r)-w_{m}(r)\right| .
\end{aligned}
$$

From (4.11) and Lemma 3.3, it is easy to see that, by passing to a suitable subsequence, we may assume that $\left\{w_{n}\right\}$ is a Cauchy sequence in $L^{2}(a, b)$ for any $a, b \in(0, \infty)$ with $a<b$. It then follows from (4.13) that $\left\{u_{n}^{\prime}\right\}$ is a Cauchy sequence in $L^{2}(a, b)$ for any $a, b \in(0, \infty)$ with $a<b$. Recalling (4.3), we now have that $\left\{u_{n}\right\}$ is a Cauchy sequence in $H^{1}(a, b)$ for all $a, b \in(0, \infty)$ with $a<b$. From this and (4.3) it follows immediately that $u_{n} \stackrel{n}{\rightarrow} u$ strongly in $H^{1}(a, b)$ for all $a, b \in(0, \infty)$ with $a<b$. By passing to a suitable (diagonal) subsequence we may henceforth assume that (4.5) holds.

Step 2. We now show that

$$
\begin{aligned}
& \int_{0}^{\infty}\left\{a\left(r, u_{n}, u_{n}^{\prime}\right)\left(u_{n}^{\prime}+\frac{u_{n}}{2 r}\right)^{2}-a\left(r, u, u^{\prime}\right)\left(u^{\prime}+\frac{u}{2 r}\right)^{2}\right\} \mathrm{d} r \\
& \quad+(\gamma(0)-\lambda) \int_{0}^{\infty}\left(u_{n}^{2}-u^{2}\right) \mathrm{d} r \rightarrow 0 \quad \text { as } n \rightarrow \infty .
\end{aligned}
$$

Since $\left\{u_{n}\right\}$ is bounded in $H_{0}^{1}(0, \infty)$, it follows from (4.1) that

$$
J^{\prime}\left(u_{n}\right) u_{n} \rightarrow 0 \quad \text { and } \quad J^{\prime}\left(u_{n}\right) u \rightarrow 0 \quad \text { as } n \rightarrow \infty
$$

Recalling (3.9), this means that

$$
\int_{0}^{\infty} a\left(r, u_{n}, u_{n}^{\prime}\right)\left[u_{n}^{2}+\left(u_{n}^{\prime}+\frac{u_{n}}{2 r}\right)^{2}\right] \mathrm{d} r-\lambda \int_{0}^{\infty} u_{n}^{2} \mathrm{~d} r \stackrel{n}{\rightarrow} 0
$$


and

$$
\int_{0}^{\infty} a\left(r, u_{n}, u_{n}^{\prime}\right)\left[u_{n} u+\left(u_{n}^{\prime}+\frac{u_{n}}{2 r}\right)\left(u^{\prime}+\frac{u}{2 r}\right)\right] \mathrm{d} r-\lambda \int_{0}^{\infty} u_{n} u \mathrm{~d} r \stackrel{n}{\rightarrow} 0 .
$$

By (4.4) and (4.5),

$$
\left\{\begin{array}{l}
a\left(r, u_{n}, u_{n}^{\prime}\right) u_{n} \stackrel{n}{\rightarrow} a\left(r, u, u^{\prime}\right) u \quad \text { a.e. on }(0, \infty) \quad \text { and } \\
a\left(r, u_{n}, u_{n}^{\prime}\right)\left(u_{n}^{\prime}+\frac{u_{n}}{2 r}\right) \stackrel{n}{\rightarrow} a\left(r, u, u^{\prime}\right)\left(u^{\prime}+\frac{u}{2 r}\right) \quad \text { a.e. on }(0, \infty)
\end{array}\right.
$$

Also, $\left\{a\left(r, u_{n}, u_{n}^{\prime}\right) u_{n}\right\}$ and $\left\{a\left(r, u_{n}, u_{n}^{\prime}\right)\left(u_{n}^{\prime}+\frac{u_{n}}{2 r}\right)\right\}$ are bounded in $L^{2}(0, \infty)$ since $\gamma(\infty) \leqslant a\left(r, u_{n}, u_{n}^{\prime}\right) \leqslant \gamma(0)$. Using Lemma 3.4, it follows from (4.16) that

$$
\int_{0}^{\infty} a\left(r, u, u^{\prime}\right)\left[u^{2}+\left(u^{\prime}+\frac{u}{2 r}\right)^{2}\right] \mathrm{d} r-\lambda \int_{0}^{\infty} u^{2} \mathrm{~d} r=0
$$

Next we show that

$$
K_{n} \rightarrow 0 \quad \text { and } \quad L_{n} \rightarrow 0 \quad \text { as } n \rightarrow \infty
$$

where

$$
K_{n}=\int_{0}^{\infty}\left|\tilde{a}\left(r, u_{n}, u_{n}^{\prime}\right) u_{n}\left(u_{n}-u\right)\right| \mathrm{d} r
$$

and

$$
L_{n}=\int_{0}^{\infty}\left|\tilde{a}\left(r, u_{n}, u_{n}^{\prime}\right) u_{n} u-\tilde{a}\left(r, u, u^{\prime}\right) u^{2}\right| \mathrm{d} r
$$

with

$$
\begin{aligned}
\tilde{a}\left(r, u_{n}, u_{n}^{\prime}\right) & =\tilde{\gamma}\left(\frac{1}{2 r}\left[u_{n}(r)^{2}+\left(u_{n}^{\prime}(r)+\frac{u_{n}(r)}{2 r}\right)^{2}\right]\right) \\
& =a\left(r, u_{n}(r), u_{n}^{\prime}(r)\right)-\gamma(0)
\end{aligned}
$$

and

$$
\begin{aligned}
\tilde{a}\left(r, u, u^{\prime}\right) & =\tilde{\gamma}\left(\frac{1}{2 r}\left[u(r)^{2}+\left(u^{\prime}(r)+\frac{u(r)}{2 r}\right)^{2}\right]\right) \\
& =a\left(r, u(r), u^{\prime}(r)\right)-\gamma(0) .
\end{aligned}
$$

As above, (4.4), (4.5) and Lemma 3.4 imply that

$$
\tilde{a}\left(r, u_{n}, u_{n}^{\prime}\right) u_{n} \stackrel{n}{\rightarrow} \tilde{a}\left(r, u, u^{\prime}\right) u \quad \text { weakly in } L^{2}(0, \infty),
$$

from which it follows immediately that $L_{n} \rightarrow 0$ as $n \rightarrow \infty$.

To deal with $K_{n}$, we consider $R>0$ and note that 
C.A. STUART, H.S. ZHOU / Ann. Inst. Henri Poincaré Anal. nonlinear 18 (2001) 69-96

$$
\begin{aligned}
K_{n} & =\int_{0}^{R}\left|\tilde{a}\left(r, u_{n}, u_{n}^{\prime}\right)\right|\left|u_{n}\left(u_{n}-u\right)\right| \mathrm{d} r+\int_{R}^{\infty}\left|\tilde{a}\left(r, u_{n}, u_{n}^{\prime}\right) u_{n}\left(u_{n}-u\right)\right| \mathrm{d} r \\
& \leqslant(\gamma(0)-\gamma(\infty)) \int_{0}^{R}\left|u_{n}\right|\left|u_{n}-u\right| \mathrm{d} r+\int_{R}^{\infty}\left|\tilde{a}\left(r, u_{n}, u_{n}^{\prime}\right) u_{n}\left(u_{n}-u\right)\right| \mathrm{d} r .
\end{aligned}
$$

By (4.3) we have that

$$
\int_{0}^{R}\left|u_{n}\right|\left|u_{n}-u\right| \mathrm{d} r \stackrel{n}{\rightarrow} 0 \text { for every } R>0 .
$$

By Lemma 2.1, there exists $\mu \in(0,1]$ and $C>0$ such that

$$
\begin{aligned}
& \int_{R}^{\infty}\left|\tilde{a}\left(r, u_{n}, u_{n}^{\prime}\right) u_{n}\left(u_{n}-u\right)\right| \mathrm{d} r \\
& \quad \leqslant C \int_{R}^{\infty}\left\{\frac{1}{2 r}\left[u_{n}^{2}+\left(u_{n}^{\prime}+\frac{u_{n}}{2 r}\right)^{2}\right]\right\}^{\mu}\left|u_{n}\left(u_{n}-u\right)\right| \mathrm{d} r \\
& \leqslant \frac{C}{2^{\mu} R^{\mu}} \int_{R}^{\infty}\left[u_{n}^{2}+\left(u_{n}^{\prime}+\frac{u_{n}}{2 r}\right)^{2}\right]^{\mu}\left|u_{n}\left(u_{n}-u\right)\right| \mathrm{d} r
\end{aligned}
$$

where

$$
\begin{aligned}
& \int_{R}^{\infty}\left[u_{n}^{2}+\left(u_{n}^{\prime}+\frac{u_{n}}{2 r}\right)^{2}\right]^{\mu}\left|u_{n}\left(u_{n}-u\right)\right| \mathrm{d} r \\
& \leqslant\left\{\begin{array}{l}
\left\{\int_{R}^{\infty}\left[u_{n}^{2}+\left(u_{n}^{\prime}+\frac{u_{n}}{2 r}\right)^{2}\right] \mathrm{d} r\right\}^{\mu}\left\{\int_{R}^{\infty}\left|u_{n}\left(u_{n}-u\right)\right|^{1 /(1-\mu)} \mathrm{d} r\right\}^{1-\mu}, \quad \text { for } \mu \in(0,1), \\
\left\{\int_{R}^{\infty}\left[u_{n}^{2}+\left(u_{n}^{\prime}+\frac{u_{n}}{2 r}\right)^{2}\right] \mathrm{d} r\right\}\left|u_{n}\right|_{\infty}\left|u_{n}-u\right|_{\infty}, \quad \text { for } \mu=1,
\end{array}\right. \\
& \leqslant\left\{\begin{array}{ll}
\left\{4\left\|u_{n}\right\|^{2}\right\}^{\mu}\left|u_{n}\right|_{2 /(1-\mu)}\left|u_{n}-u\right|_{2 /(1-\mu)} & \text { if } \mu<1, \\
4\left\|u_{n}\right\|^{2}\left|u_{n}\right|_{\infty}\left|u_{n}-u\right|_{\infty} & \text { if } \mu=1,
\end{array} \quad\right. \text { by Lemma 3.1, } \\
& \leqslant\left\{4\left\|u_{n}\right\|^{2}\right\}^{\mu}\left\|u_{n}\right\|\left\|u_{n}-u\right\| \quad \text { since }|u|_{p} \leqslant\|u\| \text { for } 2 \leqslant p \leqslant \infty \text { by (3.2). }
\end{aligned}
$$

Using (4.21) to (4.23) we see that there is a constant $D>0$ such that

$$
\limsup _{n \rightarrow \infty} K_{n} \leqslant \frac{D}{R^{\mu}} \quad \text { for all } R>0
$$

Hence $K_{n} \rightarrow 0$ as $n \rightarrow \infty$ and (4.19) is established. It follows that

$$
I_{n} \stackrel{n}{\rightarrow} 0 \quad \text { where } I_{n}=\int_{0}^{\infty}\left|\tilde{a}\left(r, u_{n}, u_{n}^{\prime}\right) u_{n}^{2}-\tilde{a}\left(r, u, u^{\prime}\right) u^{2}\right| \mathrm{d} r
$$


since $I_{n} \leqslant K_{n}+L_{n}$.

From (4.15), (4.18) and (4.24), we see that

$$
\begin{aligned}
& \int_{0}^{\infty} \gamma(0)\left(u_{n}^{2}-u^{2}\right) \mathrm{d} r+\int_{0}^{\infty}\left[a\left(r, u_{n}, u_{n}^{\prime}\right)\left(u_{n}^{\prime}+\frac{u_{n}}{2 r}\right)^{2}\right. \\
& \left.\quad-a\left(r, u, u^{\prime}\right)\left(u^{\prime}+\frac{u}{2 r}\right)^{2}\right] \mathrm{d} r-\lambda \int_{0}^{\infty}\left(u_{n}^{2}-u^{2}\right) \mathrm{d} r \rightarrow 0 \quad \text { as } n \rightarrow \infty
\end{aligned}
$$

and so (4.14) is established.

Step 3. We now complete the proof of property (c) by showing that $\left\|u_{n}-u\right\| \rightarrow 0$ as $n \rightarrow \infty$.

Indeed by (4.4), (4.17) and Fatou's Lemma,

$$
\begin{gathered}
|u|_{2}^{2} \leqslant \liminf _{n \rightarrow \infty}\left|u_{n}\right|_{2}^{2} \text { and } \\
\int_{0}^{\infty} a\left(r, u, u^{\prime}\right)\left(u^{\prime}+\frac{u}{2 r}\right)^{2} \mathrm{~d} r \leqslant \liminf _{n \rightarrow \infty} \int_{0}^{\infty} a\left(r, u_{n}, u_{n}^{\prime}\right)\left(u_{n}^{\prime}+\frac{u_{n}}{2 r}\right)^{2} \mathrm{~d} r .
\end{gathered}
$$

By (4.14), this implies that

$$
\begin{aligned}
0 & \leqslant \liminf _{n \rightarrow \infty} \int_{0}^{\infty}\left\{a\left(r, u_{n}, u_{n}^{\prime}\right)\left(u_{n}^{\prime}+\frac{u_{n}}{2 r}\right)^{2}-a\left(r, u, u^{\prime}\right)\left(u^{\prime}+\frac{u}{2 r}\right)^{2}\right\} \mathrm{d} r \\
& \leqslant \limsup _{n \rightarrow \infty} \int_{0}^{\infty}\left\{a\left(r, u_{n}, u_{n}^{\prime}\right)\left(u_{n}^{\prime}+\frac{u_{n}}{2 r}\right)^{2}-a\left(r, u, u^{\prime}\right)\left(u^{\prime}+\frac{u}{2 r}\right)^{2}\right\} \mathrm{d} r \\
& =-(\gamma(0)-\lambda) \liminf _{n \rightarrow \infty} \int_{0}^{\infty}\left[u_{n}^{2}-u^{2}\right] \mathrm{d} r \leqslant 0,
\end{aligned}
$$

since $\gamma(0)-\lambda>0$. Hence,

$$
\int_{0}^{\infty} a\left(r, u_{n}, u_{n}^{\prime}\right)\left(u_{n}^{\prime}+\frac{u_{n}}{2 r}\right)^{2} \mathrm{~d} r \stackrel{n}{\rightarrow} \int_{0}^{\infty} a\left(r, u, u^{\prime}\right)\left(u^{\prime}+\frac{u}{2 r}\right)^{2} \mathrm{~d} r
$$

and by (4.14),

$$
\left|u_{n}\right|_{2}^{2} \stackrel{n}{\rightarrow}|u|_{2}^{2} .
$$

From (4.2) and (4.27), we already have that

$$
\left|u_{n}-u\right|_{2} \rightarrow 0 \quad \text { as } n \rightarrow \infty \text {. }
$$

Furthermore

$$
\int_{0}^{\infty} a\left(r, u_{n}, u_{n}^{\prime}\right)\left[\left(u_{n}^{\prime}+\frac{u_{n}}{2 r}\right)-\left(u^{\prime}+\frac{u}{2 r}\right)\right]^{2} \mathrm{~d} r
$$




$$
=\int_{0}^{\infty} a\left(r, u_{n}, u_{n}^{\prime}\right)\left\{\left(u_{n}^{\prime}+\frac{u_{n}}{2 r}\right)^{2}-2\left(u_{n}^{\prime}+\frac{u_{n}}{2 r}\right)\left(u^{\prime}+\frac{u}{2 r}\right)+\left(u^{\prime}+\frac{u}{2 r}\right)^{2}\right\} \mathrm{d} r,
$$

where

$$
\begin{gathered}
\int_{0}^{\infty} a\left(r, u_{n}, u_{n}^{\prime}\right)\left(u_{n}^{\prime}+\frac{u_{n}}{2 r}\right)^{2} \mathrm{~d} r \stackrel{n}{\rightarrow} \int_{0}^{\infty} a\left(r, u, u^{\prime}\right)\left(u^{\prime}+\frac{u}{2 r}\right)^{2} \mathrm{~d} r \text { by (4.26), } \\
\int_{0}^{\infty} a\left(r, u_{n}, u_{n}^{\prime}\right)\left(u^{\prime}+\frac{u}{2 r}\right)^{2} \mathrm{~d} r \stackrel{n}{\rightarrow} \int_{0}^{\infty} a\left(r, u, u^{\prime}\right)\left(u^{\prime}+\frac{u}{2 r}\right)^{2} \mathrm{~d} r,
\end{gathered}
$$

by (4.4), (4.5) and dominated convergence, and

$$
\int_{0}^{\infty} a\left(r, u_{n}, u_{n}^{\prime}\right)\left(u_{n}^{\prime}+\frac{u_{n}}{2 r}\right)\left(u^{\prime}+\frac{u}{2 r}\right) \mathrm{d} r \stackrel{n}{\rightarrow} \int_{0}^{\infty} a\left(r, u, u^{\prime}\right)\left(u^{\prime}+\frac{u}{2 r}\right)^{2} \mathrm{~d} r,
$$

by (4.17) and Lemma 3.4.

Hence,

$$
\int_{0}^{\infty} a\left(r, u_{n}, u_{n}^{\prime}\right)\left[\left(u_{n}^{\prime}+\frac{u_{n}}{2 r}\right)-\left(u^{\prime}+\frac{u}{2 r}\right)\right]^{2} \mathrm{~d} r \stackrel{n}{\rightarrow} 0
$$

and since $0<\gamma(\infty) \leqslant a\left(r, u_{n}, u_{n}^{\prime}\right) \leqslant \gamma(0)$ we deduce that

$$
\int_{0}^{\infty}\left[\left(u_{n}^{\prime}+\frac{u_{n}}{2 r}\right)-\left(u^{\prime}+\frac{u}{2 r}\right)\right]^{2} \mathrm{~d} r \stackrel{n}{\rightarrow} 0
$$

Using Lemma 3.1, this implies that

$$
\int_{0}^{\infty}\left(u_{n}^{\prime}-u^{\prime}\right)^{2}+\frac{3}{4} \frac{\left(u_{n}-u\right)^{2}}{r^{2}} \mathrm{~d} r \stackrel{n}{\rightarrow} 0
$$

and so recalling (4.28), we see that $\left\|u_{n}-u\right\| \stackrel{n}{\rightarrow} 0$ as required. This completes the proof of the property (c).

Verification of (d). We fix $c>0$ and for $n \in \mathbb{N}$, we set

$$
m_{n}=\inf \left\{\left\|J^{\prime}(u)\right\|\|u\|: u \in \mathcal{S}_{n}\right\}
$$

where $\mathcal{S}_{n}=\left\{u \in H_{0}^{1}(0, \infty): c-\frac{1}{n} \leqslant J(u) \leqslant c+\frac{1}{n}\right.$ and $\left.\|u\| \geqslant n\right\}$ with $m_{n}=\infty$ if $\mathcal{S}_{n}=\emptyset$. If $J$ does not have the property (d) at this value of $c$ then $m_{n}=0$ for all $n \in \mathbb{N}$, and consequently there is a sequence $\left\{u_{n}\right\}$ such that

$$
\left\|J^{\prime}\left(u_{n}\right)\right\|\left\|u_{n}\right\|<\frac{1}{n} \quad \text { and } \quad u_{n} \in \mathcal{S}_{n}
$$


It follows that, as $n \rightarrow \infty$,

$$
J\left(u_{n}\right) \rightarrow c, \quad\left\|u_{n}\right\| \rightarrow \infty \quad \text { and } \quad\left|J^{\prime}\left(u_{n}\right) u_{n}\right|<\frac{1}{n}
$$

for all $n \in \mathbb{N}$.

We prove the property (d) by showing that the existence of such a subsequence leads to a contradiction.

From the formulae for $J$ and $J^{\prime}$, as $n \rightarrow \infty$,

$$
\int_{0}^{\infty} r \Gamma\left(\frac{1}{2 r}\left[u_{n}^{2}+\left(u_{n}^{\prime}+\frac{u_{n}}{2 r}\right)^{2}\right]\right) \mathrm{d} r-\frac{\lambda}{2} \int_{0}^{\infty} u_{n}^{2} \mathrm{~d} r \rightarrow c
$$

and

$$
-\frac{1}{n}<\int_{0}^{\infty} a\left(r, u_{n}, u_{n}^{\prime}\right)\left[u_{n}^{2}+\left(u_{n}^{\prime}+\frac{u_{n}}{2 r}\right)^{2}\right] \mathrm{d} r-\lambda \int_{0}^{\infty} u_{n}^{2} \mathrm{~d} r<\frac{1}{n}
$$

for all $n \in \mathbb{N}$. We claim that (4.30) implies that

$$
J\left(t u_{n}\right) \leqslant \frac{\left(1+t^{2}\right)}{2 n}+J\left(u_{n}\right) \text { for all } t>0 \text { and } n \in \mathbb{N} .
$$

In fact, for all $t>0$ and $n \in \mathbb{N}$,

$$
\begin{aligned}
J\left(t u_{n}\right)= & \int_{0}^{\infty} r \Gamma\left(\frac{t^{2}}{2 r}\left[u_{n}^{2}+\left(u_{n}^{\prime}+\frac{u_{n}}{2 r}\right)^{2}\right]\right) \mathrm{d} r-\frac{\lambda t^{2}}{2} \int_{0}^{\infty} u_{n}^{2} \mathrm{~d} r \\
< & \int_{0}^{\infty} r \Gamma\left(\frac{t^{2}}{2 r}\left[u_{n}^{2}+\left(u_{n}^{\prime}+\frac{u_{n}}{2 r}\right)^{2}\right]\right) \mathrm{d} r \\
& +\frac{t^{2}}{2}\left\{\frac{1}{n}-\int_{0}^{\infty} a\left(r, u_{n}, u_{n}^{\prime}\right)\left[u_{n}^{2}+\left(u_{n}^{\prime}+\frac{u_{n}}{2 r}\right)^{2}\right] \mathrm{d} r\right\} .
\end{aligned}
$$

For fixed $r$ and $n$, we set

$$
h(t)=r \Gamma\left(\frac{t^{2}}{2 r}\left[u_{n}^{2}+\left(u_{n}^{\prime}+\frac{u_{n}}{2 r}\right)^{2}\right]\right)-\frac{t^{2}}{2} a\left(r, u_{n}, u_{n}^{\prime}\right)\left[u_{n}^{2}+\left(u_{n}^{\prime}+\frac{u_{n}}{2 r}\right)^{2}\right]
$$

for $t>0$. Then

$$
\begin{aligned}
h^{\prime}(t)= & r \gamma\left(\frac{t^{2}}{2 r}\left[u_{n}^{2}+\left(u_{n}^{\prime}+\frac{u_{n}}{2 r}\right)^{2}\right]\right) \frac{2 t}{2 r}\left[u_{n}^{2}+\left(u_{n}^{\prime}+\frac{u_{n}}{2 r}\right)^{2}\right] \\
& -t a\left(r, u_{n}, u_{n}^{\prime}\right)\left[u_{n}^{2}+\left(u_{n}^{\prime}+\frac{u_{n}}{2 r}\right)^{2}\right] \\
= & t\left[u_{n}^{2}+\left(u_{n}^{\prime}+\frac{u_{n}}{2 r}\right)^{2}\right]\left\{\gamma\left(\frac{t^{2}}{2 r}\left[u_{n}^{2}+\left(u_{n}^{\prime}+\frac{u_{n}}{2 r}\right)^{2}\right]\right)\right.
\end{aligned}
$$




$$
\begin{gathered}
\left.\quad-\gamma\left(\frac{1}{2 r}\left[u_{n}^{2}+\left(u_{n}^{\prime}+\frac{u_{n}}{2 r}\right)^{2}\right]\right)\right\} \\
\left\{\begin{array}{l}
\geqslant 0 \text { for } 0<t \leqslant 1, \\
\leqslant 0 \text { for } t \geqslant 1 .
\end{array}\right.
\end{gathered}
$$

Hence, $h(t) \leqslant h(1)$ for all $t>0$ and so (4.32) yields

$$
\begin{aligned}
J\left(t u_{n}\right) \leqslant & \frac{t^{2}}{2 n}+\int_{0}^{\infty} h(t) \mathrm{d} r \leqslant \frac{t^{2}}{2 n}+\int_{0}^{\infty} h(1) \mathrm{d} r \\
= & \frac{t^{2}}{2 n}+\int_{0}^{\infty} r \Gamma\left(\frac{1}{2 r}\left[u_{n}^{2}+\left(u_{n}^{\prime}+\frac{u_{n}}{2 r}\right)^{2}\right]\right) \\
& -\frac{1}{2} a\left(r, u_{n}, u_{n}^{\prime}\right)\left[u_{n}^{2}+\left(u_{n}^{\prime}+\frac{u_{n}}{2 r}\right)^{2}\right] \mathrm{d} r .
\end{aligned}
$$

But, again from (4.30),

$$
\begin{aligned}
J\left(u_{n}\right) \geqslant & \int_{0}^{\infty} r \Gamma\left(\frac{1}{2 r}\left[u_{n}^{2}+\left(u_{n}^{\prime}+\frac{u_{n}}{2 r}\right)^{2}\right]\right) \mathrm{d} r \\
& -\frac{1}{2}\left\{\frac{1}{n}+\int_{0}^{\infty} a\left(r, u_{n}, u_{n}^{\prime}\right)\left[u_{n}^{2}+\left(u_{n}^{\prime}+\frac{u_{n}}{2 r}\right)^{2}\right]\right\} \mathrm{d} r
\end{aligned}
$$

so that

$$
\begin{aligned}
& \int_{0}^{\infty} r \Gamma\left(\frac{1}{2 r}\left[u_{n}^{2}+\left(u_{n}^{\prime}+\frac{u_{n}}{2 r}\right)^{2}\right]\right)-\frac{1}{2} a\left(r, u_{n}, u_{n}^{\prime}\right)\left[u_{n}^{2}+\left(u_{n}^{\prime}+\frac{u_{n}}{2 r}\right)^{2}\right] \mathrm{d} r \\
& \quad \leqslant \frac{1}{2 n}+J\left(u_{n}\right) \quad \text { for all } n \in \mathbb{N} .
\end{aligned}
$$

Combining (4.33) and (4.34), we see that the claim (4.31) has been established.

Now we set

$$
d=\frac{2 \sqrt{c}}{\left[\min \left\{\gamma(\infty), \frac{\gamma(0)-\lambda}{2}\right\}\right]^{1 / 2}}, \quad t_{n}=\frac{d}{\left\|u_{n}\right\|} \quad \text { and } \quad w_{n}=t_{n} u_{n} .
$$

Clearly, the sequence $\left\{w_{n}\right\}$ is bounded in $H_{0}^{1}(0, \infty)$ and so, by passing to a subsequence, we can suppose that $w_{n} \rightarrow w$ weakly in $H_{0}^{1}(0, \infty)$ for some element $w \in H_{0}^{1}(0, \infty)$. We obtain the desired contradiction by showing that there can be no such element $w$. We do this in three steps. First we show that $w \neq 0$. Then we show that $w$ must satisfy a certain linear differential equation. Finally we show that this equation cannot have a nonzero solution in $H_{0}^{1}(0, \infty)$.

Step 1. Suppose that $w=0$. In particular $w_{n} \rightarrow 0$ uniformly on compact subsets of $[0, \infty)$. As in the proof of part (a) of Proposition 4.2, we note that the concavity of $\Gamma$ implies that 


$$
\begin{aligned}
J\left(w_{n}\right) & =\int_{0}^{\infty}\left\{r \Gamma\left(\frac{1}{2 r}\left[w_{n}^{2}+\left(w_{n}^{\prime}+\frac{w_{n}}{2 r}\right)^{2}\right]\right)-\frac{\lambda}{2} w_{n}^{2}\right\} \mathrm{d} r \\
& \geqslant \int_{0}^{\infty}\left\{\frac{r}{2}\left[\Gamma\left(\frac{w_{n}^{2}}{r}\right)+\frac{\gamma(\infty)}{r}\left(w_{n}^{\prime}+\frac{w_{n}}{2 r}\right)^{2}\right]-\frac{\lambda}{2} w_{n}^{2}\right\} \mathrm{d} r \\
& =\frac{\gamma(\infty)}{2} \int_{0}^{\infty}\left(w_{n}^{\prime}+\frac{w_{n}}{2 r}\right)^{2} d r+\frac{1}{2} \int_{0}^{\infty}\left\{r \Gamma\left(\frac{w_{n}^{2}}{r}\right)-\frac{\lambda}{2} w_{n}^{2}\right\} \mathrm{d} r .
\end{aligned}
$$

Let $\varepsilon=(\gamma(0)-\lambda) / 2$. Then there exists $\delta>0$ such that $\Gamma(t) \geqslant(\gamma(0)-\varepsilon) t$ for all $t \in[0, \delta]$. Setting $R(\varepsilon)=d^{2} / \delta$, we have that $w_{n}^{2}(r) / r \leqslant d^{2} / R(\varepsilon) \leqslant \delta$ for all $r \geqslant R(\varepsilon)$, and so

$$
\int_{R(\varepsilon)}^{\infty} r \Gamma\left(\frac{w_{n}^{2}}{r}\right) \mathrm{d} r \geqslant \int_{R(\varepsilon)}^{\infty} r[\gamma(0)-\varepsilon] \frac{w_{n}^{2}}{r} \mathrm{~d} r
$$

for all $n$. Hence

$$
\begin{aligned}
\int_{0}^{\infty} r \Gamma\left(\frac{w_{n}^{2}}{r}\right) \mathrm{d} r & \geqslant \int_{0}^{R(\varepsilon)} r \gamma(\infty) \frac{w_{n}^{2}}{r} \mathrm{~d} r+\int_{R(\varepsilon)}^{\infty}[\gamma(0)-\varepsilon] w_{n}^{2} \mathrm{~d} r \\
& =\int_{0}^{\infty}[\gamma(0)-\varepsilon] w_{n}^{2} \mathrm{~d} r+\int_{0}^{R(\varepsilon)}[\gamma(\infty)-\gamma(0)+\varepsilon] w_{n}^{2} \mathrm{~d} r \\
& =[\gamma(0)-\varepsilon] \int_{0}^{\infty} w_{n}^{2} \mathrm{~d} r+\mathrm{o}(1)
\end{aligned}
$$

since $w_{n} \rightarrow 0$ uniformly on $[0, R(\varepsilon)]$ as $n \rightarrow \infty$. Thus we see that

$$
\begin{aligned}
J\left(w_{n}\right) & \geqslant \frac{\gamma(\infty)}{2} \int_{0}^{\infty}\left(w_{n}^{\prime}\right)^{2} \mathrm{~d} r+\frac{1}{2}[\gamma(0)-\varepsilon-\lambda] \int_{0}^{\infty} w_{n}^{2} \mathrm{~d} r+\mathrm{o}(1) \\
& =\frac{\gamma(\infty)}{2} \int_{0}^{\infty}\left(w_{n}^{\prime}\right)^{2} \mathrm{~d} r+\frac{1}{4}[\gamma(0)-\lambda] \int_{0}^{\infty} w_{n}^{2} \mathrm{~d} r+\mathrm{o}(1) \\
& \geqslant \frac{1}{2} \min \left\{\gamma(\infty), \frac{1}{2}[\gamma(0)-\lambda]\right\}\left\|w_{n}\right\|^{2}+\mathrm{o}(1) \\
& =2 c+o(1)
\end{aligned}
$$

by the definition of $d$ and $w_{n}$.

On the other hand we have already shown in (4.31) that

$$
\begin{aligned}
J\left(w_{n}\right) & =J\left(t_{n} u_{n}\right) \leqslant \frac{\left(1+t_{n}^{2}\right)}{2 n}+J\left(u_{n}\right) \text { for all } t_{n}>0 \text { and } n \in \mathbb{N} \\
& =c+o(1),
\end{aligned}
$$


where as before o(1) represents any quantity which tends to zero as $n \rightarrow \infty$. Combining these inequalities we see find that

$$
2 c+\mathrm{o}(1) \leqslant c+\mathrm{o}(1)
$$

contradicting the fact that $c>0$. This proves that $w \neq 0$.

Step 2. At this point our aim is to show that for all $\varphi \in C_{0}^{\infty}((0, \infty))$,

$$
\int_{0}^{\infty} \gamma(\infty)\left\{w \varphi+\left(w^{\prime}+\frac{w}{2 r}\right)\left(\varphi^{\prime}+\frac{\varphi}{2 r}\right)\right\}-\lambda w \varphi \mathrm{d} r=0 .
$$

We begin by recalling that $\left\|J^{\prime}\left(u_{n}\right)\right\| \rightarrow 0$ and $t_{n} \rightarrow 0$. Hence, for all $\varphi \in C_{0}^{\infty}((0, \infty))$,

$$
\begin{aligned}
& \int_{0}^{\infty} a\left(r, u_{n}, u_{n}^{\prime}\right)\left\{w_{n} \varphi+\left(w_{n}^{\prime}+\frac{w_{n}}{2 r}\right)\left(\varphi^{\prime}+\frac{\varphi}{2 r}\right)\right\}-\lambda w_{n} \varphi \mathrm{d} r \\
& \quad=t_{n} J^{\prime}\left(u_{n}\right) \varphi \rightarrow 0 \quad \text { as } n \rightarrow \infty .
\end{aligned}
$$

To complete this step we show that, as $n \rightarrow \infty$,

$$
\text { (i) } \int_{0}^{\infty}\left\{a\left(r, u_{n}, u_{n}^{\prime}\right)-\lambda\right\} w_{n} \varphi \mathrm{d} r \rightarrow \int_{0}^{\infty}\{\gamma(\infty)-\lambda\} w \varphi \mathrm{d} r
$$

and

(ii) $\int_{0}^{\infty} a\left(r, u_{n}, u_{n}^{\prime}\right)\left(w_{n}^{\prime}+\frac{w_{n}}{2 r}\right)\left(\varphi^{\prime}+\frac{\varphi}{2 r}\right) \mathrm{d} r \rightarrow \int_{0}^{\infty} \gamma(\infty)\left(w^{\prime}+\frac{w}{2 r}\right)\left(\varphi^{\prime}+\frac{\varphi}{2 r}\right) \mathrm{d} r$.

We fix $\varphi \in C_{0}^{\infty}((0, \infty))$ and choose $k, l$ such that supp $\varphi \subset[k, l] \subset(0, \infty)$. For $m \in \mathbb{N}$, let

$$
A(n, m)=\left\{r \in[k, l]: w_{n}(r)^{2}+\left[w_{n}^{\prime}(r)+\frac{w_{n}(r)}{2 r}\right]^{2} \leqslant \frac{1}{m}\right\}
$$

and let $B(n, m)=[k, l] \backslash A(n, m)$.

There exists $s_{m}>0$ such that $|\gamma(s)-\gamma(\infty)| \leqslant 1 / m$ for all $s \geqslant s_{m}$. But

$$
a\left(r, u_{n}, u_{n}^{\prime}\right)=\gamma\left(\frac{1}{2 r t_{n}^{2}}\left[w_{n}(r)^{2}+\left[w_{n}^{\prime}(r)+\frac{w_{n}(r)}{2 r}\right]^{2}\right]\right),
$$

where

$$
\frac{1}{2 r t_{n}^{2}}\left[w_{n}(r)^{2}+\left[w_{n}^{\prime}(r)+\frac{w_{n}(r)}{2 r}\right]^{2}\right] \geqslant \frac{1}{2 l t_{n}^{2} m}
$$

for all $r \in B(n, m)$. Hence, since $t_{n} \rightarrow 0$, there exists $n(m) \in \mathbb{N}$ such that

$$
\frac{1}{2 r t_{n}^{2}}\left[w_{n}(r)^{2}+\left[w_{n}^{\prime}(r)+\frac{w_{n}(r)}{2 r}\right]^{2}\right] \geqslant s_{m} \quad \text { for all } n \geqslant n(m)
$$


and so

$$
\left|a\left(r, u_{n}, u_{n}^{\prime}\right)-\gamma(\infty)\right| \leqslant \frac{1}{m}
$$

for all $r \in B(n, m)$ provided that $n \geqslant n(m)$. Hence

$$
\begin{aligned}
\int_{0}^{\infty}\left|a\left(r, u_{n}, u_{n}^{\prime}\right)-\gamma(\infty)\right|\left|w_{n}\right||\varphi| \mathrm{d} r & =\left(\int_{A(n, m)}+\int_{B(n, m)}\right)\left|a\left(r, u_{n}, u_{n}^{\prime}\right)-\gamma(\infty)\right|\left|w_{n}\right||\varphi| \mathrm{d} r \\
& \leqslant \frac{[\gamma(0)-\gamma(\infty)]}{\sqrt{m}} \int_{A(n, m)}|\varphi| \mathrm{d} r+\frac{1}{m} \int_{B(n, m)}\left|w_{n}\right||\varphi| \mathrm{d} r
\end{aligned}
$$

provided that $n \geqslant n(m)$. Recalling that $\left|w_{n}\right|_{\infty} \leqslant\left\|w_{n}\right\|=d$, we see that

$$
\lim \sup _{n \rightarrow \infty} \int_{0}^{\infty}\left|a\left(r, u_{n}, u_{n}^{\prime}\right)-\gamma(\infty)\right|\left|w_{n}\right||\varphi| \mathrm{d} r \leqslant\left\{\frac{[\gamma(0)-\gamma(\infty)]}{\sqrt{m}}+\frac{d}{m}\right\} \int_{0}^{\infty}|\varphi| \mathrm{d} r
$$

for all $m \in \mathbb{N}$. Thus

$$
\lim _{n \rightarrow \infty} \int_{0}^{\infty}\left|a\left(r, u_{n}, u_{n}^{\prime}\right)-\gamma(\infty)\right|\left|w_{n}\right||\varphi| \mathrm{d} r=0
$$

and (i) follows from the weak convergence of $w_{n}$ to $w$ in $H_{0}^{1}(0, \infty)$.

Similarly,

$$
\begin{aligned}
& \int_{0}^{\infty}\left|a\left(r, u_{n}, u_{n}^{\prime}\right)-\gamma(\infty)\right|\left|\left(w_{n}^{\prime}+\frac{w_{n}}{2 r}\right)\right|\left|\left(\varphi^{\prime}+\frac{\varphi}{2 r}\right)\right| \mathrm{d} r \\
& \quad=\left(\int_{A(n, m)}+\int_{B(n, m)}\right)\left|a\left(r, u_{n}, u_{n}^{\prime}\right)-\gamma(\infty)\right|\left|\left(w_{n}^{\prime}+\frac{w_{n}}{2 r}\right)\right|\left|\left(\varphi^{\prime}+\frac{\varphi}{2 r}\right)\right| \mathrm{d} r \\
& \quad \leqslant \frac{[\gamma(0)-\gamma(\infty)]}{\sqrt{m}} \int_{A(n, m)}\left|\left(\varphi^{\prime}+\frac{\varphi}{2 r}\right)\right| \mathrm{d} r+\frac{1}{m} \int_{B(n, m)}\left|\left(w_{n}^{\prime}+\frac{w_{n}}{2 r}\right)\right|\left|\left(\varphi^{\prime}+\frac{\varphi}{2 r}\right)\right| \mathrm{d} r
\end{aligned}
$$

provided that $n \geqslant n(m)$. Hence

$$
\begin{aligned}
& \lim \sup _{n \rightarrow \infty} \int_{0}^{\infty}\left|a\left(r, u_{n}, u_{n}^{\prime}\right)-\gamma(\infty)\right|\left|\left(w_{n}^{\prime}+\frac{w_{n}}{2 r}\right)\right|\left|\left(\varphi^{\prime}+\frac{\varphi}{2 r}\right)\right| \mathrm{d} r \\
& \leqslant \lim \sup _{n \rightarrow \infty} \frac{[\gamma(0)-\gamma(\infty)]}{\sqrt{m}} \int_{0}^{\infty}\left|\left(\varphi^{\prime}+\frac{\varphi}{2 r}\right)\right| \mathrm{d} r+\frac{4}{m}\left|w_{n}^{\prime}\right|_{2}\left|\varphi_{n}^{\prime}\right|_{2} \\
& \leqslant \frac{[\gamma(0)-\gamma(\infty)]}{\sqrt{m}} \int_{0}^{\infty}\left|\left(\varphi^{\prime}+\frac{\varphi}{2 r}\right)\right| \mathrm{d} r+\frac{4 d}{m}\left|\varphi_{n}^{\prime}\right|_{2}
\end{aligned}
$$


by (3.4) and (3.6) for all $m \in \mathbb{N}$. Thus

$$
\lim _{n \rightarrow \infty} \int_{0}^{\infty}\left|a\left(r, u_{n}, u_{n}^{\prime}\right)-\gamma(\infty)\right|\left|\left(w_{n}^{\prime}+\frac{w_{n}}{2 r}\right)\right|\left|\left(\varphi^{\prime}+\frac{\varphi}{2 r}\right)\right| \mathrm{d} r=0
$$

and (ii) follows from the weak convergence of $w_{n}$ to $w$ in $H_{0}^{1}(0, \infty)$.

Step 3. By step 2 we know that for all $\varphi \in C_{0}^{\infty}((0, \infty))$,

$$
\int_{0}^{\infty}\left\{w \varphi+\left(w^{\prime}+\frac{w}{2 r}\right)\left(\varphi^{\prime}+\frac{\varphi}{2 r}\right)\right\}-\mu w \varphi \mathrm{d} r=0
$$

where $\mu=\lambda / \gamma(\infty)>1$ and $w \in H_{0}^{1}(0, \infty)$. It follows that $\left(w^{\prime}+\frac{w}{2 r}\right)$ has a weak derivative on $(0, \infty)$ and that

$$
\left(w^{\prime}+\frac{w}{2 r}\right)^{\prime}=(1-\mu) w+\frac{1}{2 r}\left(w^{\prime}+\frac{w}{2 r}\right) .
$$

This equation simplifies to

$$
w^{\prime \prime}-\frac{3}{4 r^{2}} w=(1-\mu) w
$$

which is a form of Bessel's equation having two linearly independent solutions which can be expressed as

$$
w_{1}(r)=\sqrt{r} J_{1}(\sqrt{\mu-1} r) \text { and } w_{2}(r)=\sqrt{r} Y_{1}(\sqrt{\mu-1} r),
$$

where $J_{1}$ and $Y_{1}$ denote the usual Bessel functions of order one of the first and second kind, respectively. (Here we use the standard notation as in [6], for example.) Hence there are constants $A$ and $B$ such that

$$
w(r)=A w_{1}(r)+B w_{2}(r) \text { for } r>0 .
$$

But, from the properties of $J_{1}$ and $Y_{1}$ (see Sections 5.16.1 and 5.16.2 of [6]), it follows that

$$
\lim _{r \rightarrow 0} w_{1}(r)=0 \quad \text { and } \quad \lim _{r \rightarrow 0} w_{2}(r)=\infty
$$

and so we must have $B=0$. On the other hand,

$$
\lim _{r \rightarrow \infty} \frac{w_{1}(r)}{\cos \left(\sqrt{\mu-1} r-\frac{3}{4} \pi\right)}=\sqrt{\frac{2}{\pi \sqrt{(\mu-1)}}}
$$

which shows that $w_{1} \notin L^{2}(0, \infty)$. But $w \in H_{0}^{1}(0, \infty) \subset L^{2}(0, \infty)$ so we must have $A=0$. Thus we find that $w \equiv 0$, contradicting what was proved in step 1 .

Thus we see that the assumption that $m_{n}=0$ for all $n \in \mathbb{N}$ leads to a contradiction and this in turn proves that the property (d) of Proposition 4.2 is satisfied.

This completes the proof of Theorem 4.1. 


\section{Acknowledgement}

This work was supported by Swiss National Science Foundation (Project number 2046937.96). The second author would like to thank the Swiss NSF for supporting his stay at the EPFL and also the Department of Mathematics at the EPFL for its hospitality.

\section{REFERENCES}

[1] Bartolo P., Benci V., Fortunato D., Abstract critical point theorems and applications to some nonlinear problems with strong resonance at infinity, Nonlinear Anal. TMA 7 (9) (1983) 981-1012.

[2] Chen Y., TE and TM families of self-trapped beams, IEEE J. Quantum Electronics 27 (1991) 1236-1241.

[3] Chen Y., Snyder A.W., TM-type self-guided beams with circular cross-section, Electr. Lett. 27 (1991) 564-566.

[4] Jeanjean L., On the existence of bounded Palais-Smale sequences and application to a Landesman-Lazer type problem, Proc. Royal Soc. Edinburgh, Ser. A 129A (1999) 787809.

[5] John O., Stuart C.A., Guidance properties of a cylindrical defocusing waveguide, Comm. Math. Univ. Carolinae 35 (1994) 653-673.

[6] Lebedev N.N., Special Functions and their Applications, Dover, 1972.

[7] Rabinowitz P.H., Minimax Methods in Critical Point Theory with Applications to Differential Equations, CBMS Reg. Conf. Ser. in Math., No. 65, Amer. Math. Soc., Providence, RI, 1986.

[8] Ruppen H.-J., Multiple cylindrical TE-Modes for a homogeneous self-focusing dielectric, Nonlinear World 2 (1995) 387-418.

[9] Ruppen H.-J., Multiple cylindrical TM-Modes in a homogeneous self-focusing dielectric, J. Differential Equations 201 (1997) 112-123.

[10] Showalter R.E., Monotone Operators in Banach Space and Nonlinear Partial Differential Equations, Mathematical Surveys and Monographs (AMS), Vol. 49, 1997.

[11] Stuart C.A., Self-trapping of an electromagnetic field and bifurcation from the essential spectrum, Arch. Rat. Mech. Anal. 113 (1991) 65-96.

[12] Stuart C.A., Cylindrical TM-modes in a homogeneous self-focusing dielectric, Math. Models Methods Appl. Sci. 6 (1996) 977-1008.

[13] Stuart C.A., Magnetic field wave equations for TM-modes in nonlinear optical waveguides, in: Caristi, Mitidieri (Eds.), Reaction Diffusion Systems, Marcel Dekker, 1997.

[14] Stuart C.A., Zhou H.S., A variational problem related to self-trapping of an electromagnetic field, Math. Methods Appl. Sci. 19 (1996) 1397-1407. 